\title{
PLANTAS VASCULARES ENDÉMICAS DE COAHUILA Y ALGUNAS ÁREAS ADYACENTES, MÉXICO
}

\author{
José Á. Villarreal-Quintanilla y Juan A. Encina-Domínguez \\ Departamento de Botánica, Universidad Autónoma Agraria Antonio Narro \\ Buenavista 25315 Saltillo, Coahuila
}

\begin{abstract}
RESUMEN
Se revisa la distribución de especies de plantas endémicas tomando como base el concepto de Megacoahuila, que amplía la superficie del estado en más de $100 \%$. La región ofrece variación climática y fisiográfica que da lugar a la presencia de al menos 13 tipos de vegetación de los que el matorral desértico es el dominante. Se presenta un listado de 350 especies y taxa infraespecíficos. Esta cifra representa $11.2 \%$ del total de la flora. Los tipos de vegetación con mayor incidencia de elementos con distribución restringida son los matorrales micrófilo y rosetófilo y la localidad con el mayor número es Cuatro Ciénegas. Se sugiere la creación de reservas bióticas en las áreas particularmente ricas en endemismos.
\end{abstract}

Palabras clave: Coahuila, endemismo, Megacoahuila, México, plantas vasculares.

\begin{abstract}
A review is made of the endemic plants following the Megacoahuila concept, that increases the state area for more than $100 \%$. The region has climatic and physiographic variation that leads to the development of at least 13 main vegetation types, the desert shrubland being the dominant one. A total of 350 species and infraspecific taxa are listed, representing $11.2 \%$ of the total flora. The desert shrubland (matorrales micrófilo y rosetófilo) is the vegetation with the highest endemism and the Cuatro Cienegas basin the richest locality. The areas with highest endemism are suggested to be considered biotic reserves.
\end{abstract}

Key words: Coahuila, endemism, Megacoahuila, Mexico, vascular plants.

\section{INTRODUCCIÓN}

México es reconocido como un lugar con alta biodiversidad (Gómez-Pompa et al., 1994). De acuerdo con Rzedowski (1991b), la flora fanerogámica endémica 
del país se estima en 9,300 especies, mientras que para el concepto ampliado de Megaméxico 3 se calcula en 12,900 especies, lo que implica un porcentaje aproximado del 52 y el 72 del total de la misma respectivamente. Los endemismos, en particular a nivel de especie, son frecuentes sobre todo en regiones templadas y subhúmedas y zonas áridas y semiáridas (Rzedowski, 1991a).

En el estado de Coahuila se presenta gran variedad de condiciones fisiográficas, climáticas y edáficas, factores que han dado lugar a una significativa diversidad de tipos de vegetación y de flora. Esta última se estima en aproximadamente 3,100 especies y taxa infraespecíficos de plantas vasculares (Villarreal, 2001). El aislamiento de numerosos sistemas montañosos o enclaves orográficos de Coahuila y la presencia de cuencas endorréicas donde se registran condiciones edáficas especiales, contribuyen a incrementar el número de elementos de distribución restringida (Villarreal et al., 1996). Johnston (1941) y Rzedowski (1991b) señalan que entre los endemismos edáficos destacan las gipsófitas, con una historia evolutiva antigua, con frecuencia concentrados en áreas que han funcionado como refugios durante épocas de clima cambiante del Terciario y Cuaternario.

La mayor parte del territorio coahuilense está incluida en el Desierto Chihuahuense y de acuerdo con Henrickson y Johnston (inédito), contribuye más que cualquier otro estado al número de especies endémicas para esta gran zona árida. En el estado se presenta la máxima concentración (29 especies) de cactáceas amenazadas dentro del Desierto Chihuahuense (Hernández y Godínez, 1994); y en él se localiza un importante centro de diversidad de la tribu Heliantheae (Asteraceae) para México, con 19 especies endémicas distribuidas principalmente en las cuencas endorréicas del centro y de la Sierra Madre Oriental en el sureste del estado (Villaseñor, 1991).

La riqueza florística de Coahuila ha sido señalada por diversos autores, entre los que se encuentran: Pinkava (1984), en su estudio sobre la vegetación y flora del Bolsón de Cuatro Ciénegas; Vásquez et al. (1989), en relación a los pastizales del rancho experimental ganadero Los Ángeles, municipio de Saltillo; McDonald (1990), con referencia a la flora alpina y subalpina del noreste de México; Elizondo et al. (1990), en su enumeración de las cactáceas vulnerables y en peligro de extinción; Rodríguez et al. (1994), quienes describieron la vegetación y flora de la Sierra de Parras; Villarreal (1994), autor del trabajo sobre la vegetación y flora de la Sierra de La Paila; Hinton y Hinton (1995), en su lista de la flora del sur de Nuevo León y áreas adyacentes de Coahuila; Phipps (1997), en el trabajo sobre el género Crataegus en el norte de México, y Henrickson y Johnston (inédito) en la flora del Desierto Chihuahuense, entre otros.

Un taxon es endémico a un área cuando todas sus poblaciones presentan distribución restringida a la misma; la cual puede variar en extensión. Aunque es evidente que la repartición de los organismos no necesariamente coincide con las 
entidades administrativas, la elección de áreas de orden estatal es conveniente, ya que las medidas de conservación se circunscriben comúnmente a las delimitaciones políticas más que a las áreas naturales (Villaseñor et al., 1998). Sin embargo, con el propósito de proporcionar a las especies endémicas un marco geográfico de referencia más natural, y no restringido estrictamente a los límites del estado, se adopta el concepto de Megacoahuila (Villarreal et al., 1996).

\section{ÁREA DE ESTUDIO}

En el presente trabajo se consideran endémicas las especies presentes en Coahuila y que pueden extender su distribución a Megacoahuila. Este último concepto implica extender los límites del estudio a las siguientes entidades vecinas: del estado de Texas se incluye casi toda la región de Trans-Pecos, con las Montañas Davis, Chinati, Glass y Chisos; de la región del Edwards Plateau los condados Terrell, Crockett y Val Verde y el área del cañón del Río Bravo; de Chihuahua, parte de los municipios de Juárez, Guadalupe, Coyamé, Ojinaga, Manuel Benavides, Camargo y Jiménez, que incluyen las sierras calizas de Santa Elena, Hechiceros, El Diablo, Almagre, Púlpito, Grande, del Pino y de La Lágrima, además de una gran parte del Bolsón de Mapimí; de Durango una porción de territorio de los municipios colindantes abarcando extensas áreas de los de Mapimí, Nazas y Cuencamé, que incluye el complejo de las Sierras El Rosario y Los Álamos; de Zacatecas, gran parte de los municipios limítrofes de Melchor Ocampo, Concepción del Oro y un sector de Mazapil; de San Luis Potosí los valles halófilos localizados en Vanegas y Cedral; de Nuevo León, la porción de los municipios de Anáhuac, Lampazos, Villa Aldama, Bustamante, Mina, García, Santa Catarina y Monterrey, en los que se encuentran las Sierras de Lampazos, Gomas, Mina y El Fraile, además de valles salinos; adicionalmente, del sureste del mencionado estado se incluyen los municipios de Santiago, Rayones, Galeana e Iturbide, correspondiendo a la parte de la Sierra Madre Oriental que se extiende hasta el cerro de El Potosí y a los valles con suelos salinoyesosos; y de Tamaulipas, una porción del extremo norte correspondiente al municipio de Nuevo Laredo (Fig. 1).

En el área confluyen tres provincias fisiográficas: la Planicie Costera del Golfo, la Provincia de la Sierra Madre Oriental y el Altiplano Mexicano. El intervalo altitudinal oscila desde $190 \mathrm{~m}$ en la primera, hasta $3,450 \mathrm{~m}$ en las cimas montañosas ubicadas en el sureste del estado. El clima, de acuerdo con García (1987), varía mayormente entre $\mathrm{BS}_{0}$ y BWh, siendo éstos continentales y secos, predominantes en los bolsones coahuilenses. El tipo $\mathrm{Cw}$ o templado y subhúmedo se presenta en las porciones más altas de las sierras. La parte nororiental del área se ubica al este de la barrera orográfica de la Sierra Madre Oriental y así los climas predominantes 


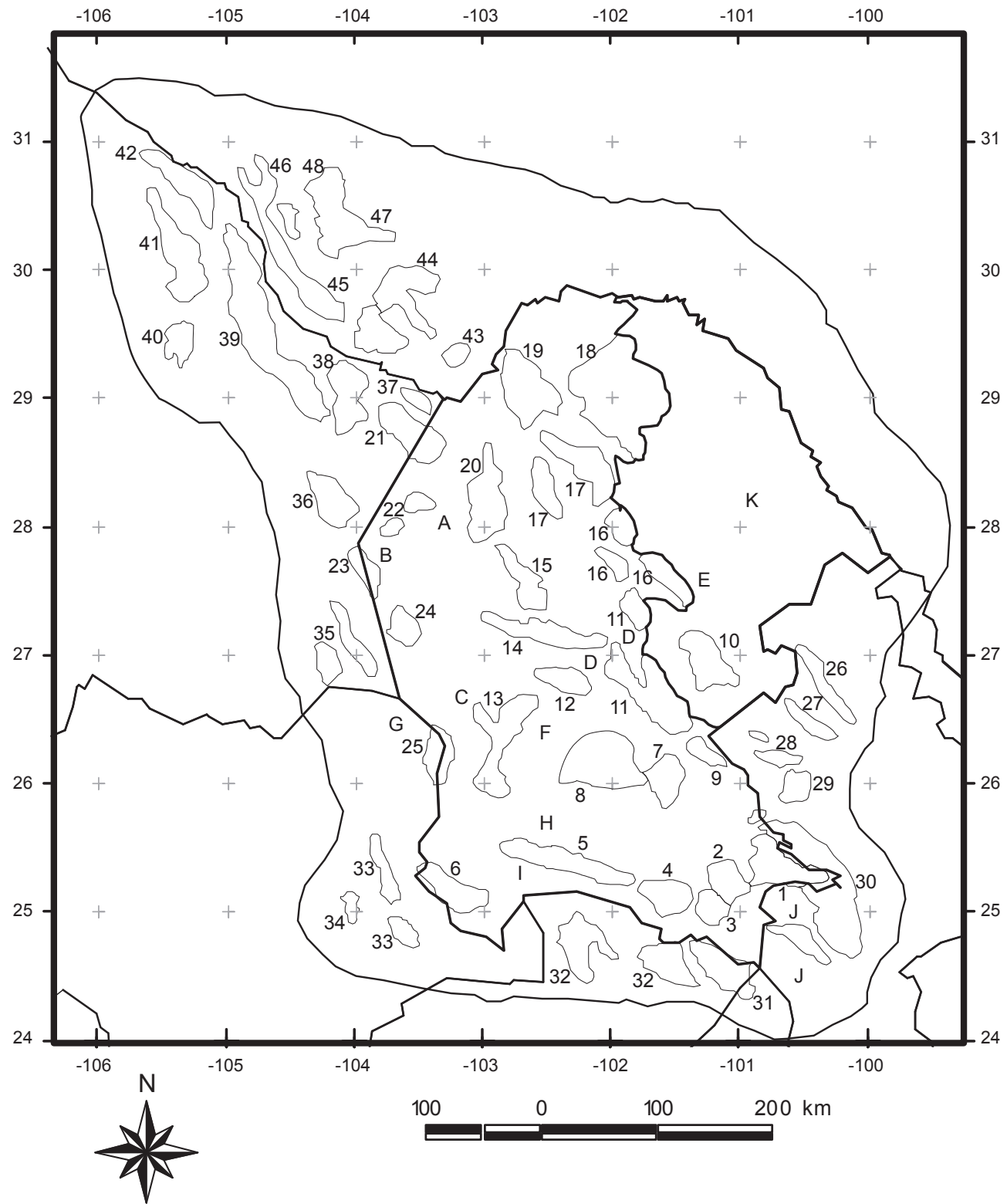

Fig. 1. La región de Megacoahuila y la ubicación de los principales elementos de su fisiografía. La explicación de los símbolos del mapa se encuentra en la siguiente página. No todas las sierras, lagunas, bolsones y planicies mencionadas en el texto del Apéndice 1 se encuentran representadas en esta cartografía. 
I. Macizos montañosos.

\section{COAHUILA}

1. Sierra de Arteaga

2. Sierra de Zapalinamé

3. Sierra de Carneros

4. Sierra de La Concordia

5. Sierra de Parras

6. Sierra de Jimulco

7. Sierra de La Paila

8. Sierra de Los Alamitos

9. Sierra de La Gavia

10. Sierra de La Gloria

11. Sierra de San Marcos

12. Sierra de La Fragua

13. Sierra de Las Delicias

14. Sierra de La Madera

15. Sierra del Fuste

16. Sierra Santa Rosa

17. Sierra La Encantada

18. Serranías del Burro

19. Sierra del Carmen

20. Sierra del Pino

21. Sierra Hechiceros

22. Sierra Cruces

23. Sierra de Almagre

24. Sierra Mojada

25. Sierra de Tlahualilo

\section{NUEVO LEÓN}

26. Sierra de Lampazos

27. Sierra de Gomas

28. Sierra de Mina

II. Lagunas, bolsones y planicies.
A. Laguna del Guaje
B. Laguna del Jaco
C. Laguna del Rey
D. Valle de Cuatro Ciénegas
E. Valle de Hermanas
F. Valles del Sobaco y El Hundido
G. Bolsón de Mapimí

29. Sierra de El Fraile

30. Sierra Madre Oriental (hasta el cerro El Potosí)

\section{ZACATECAS}

31. Sierra de Astillero

32. Sierras al sur de Coahuila y norte de Zacatecas

DURANGO

33. Sierra de El Rosario

34. Sierra de Los Álamos

CHIHUAHUA

35. Sierra de El Diablo

36. Sierra Encinillas

37. Sierra de Santa Elena

38. Sierra Mulato

39. Sierra Grande

40. Sierra del Pulpito

41. Sierra de La Lágrima

42. Sierra de El Pino

\section{TEXAS}

43. Montañas Chisos

44. Montañas Glass

45. Montañas Chinati

46. Montañas Sierra Vieja

47. Montañas Davis

48. Montañas Apache
H. Laguna de Mayrán

I. Viesca

J. Áreas salino-yesosas del sureste de Coahuila, así como de porciones contiguas de Nuevo León y San Luis Potosí

K. Planicie Costera del Golfo 
en estos territorios son más húmedos y cálidos y la influencia marítima más notable. La precipitación es escasa durante la mayor parte del año, su promedio anual varía desde 146 hasta $632 \mathrm{~mm}$ (García, 1987), pero en las porciones altas de las montañas el valor es superior a $650 \mathrm{~mm}$. La temporada con mayor precipitación es de julio a octubre. La temperatura es sumamente variable, desde $-8^{\circ} \mathrm{C}$ en la época invernal en las partes altas, hasta $38^{\circ} \mathrm{C}$ en el verano en las porciones de menor altitud. La humedad atmosférica en la mayor parte del territorio es baja y la evaporación elevada. En general el clima es extremoso, con veranos muy calurosos y los inviernos fríos.

El patrón edáfico también es diverso y está determinado por las condiciones topográficas, geomórficas y climáticas de la zona. Los xerosoles y yermosoles son suelos típicos de climas secos o semisecos. En los macizos montañosos se presentan los litosoles y regosoles calcáricos.

La cubierta vegetal, según Villarreal y Valdés (1992-1993), está representada por 13 tipos de vegetación. El matorral desértico chihuahuense es la comunidad más característica, comprende: a) el matorral micrófilo en suelos aluviales profundos, donde las especies más frecuentes son Larrea tridentata, Flourensia cernua, Parthenium incanum y Prosopis glandulosa; b) el matorral rosetófilo en laderas rocosas de lomeríos y sierras, integrado principalmente por Agave lechuguilla y Dasylirion cedrosanum; donde se presenta un amplia diversidad de cactáceas; c) los matorrales de halófitas y gipsófitas, ocupan fondos de cuencas aisladas con drenaje interno, cuya presencia está determinada por condiciones edáficas locales.

El matorral tamaulipeco se distribuye al noreste del área, se localiza en la provincia de la Planicie Costera del Golfo, su asociación más frecuente es la de Acacia rigidula, Leucophyllum frutescens y Prosopis glandulosa. El matorral submontano, localizado en las laderas y cañones de las sierras, presenta elementos del género Rhus, Acacia, Cercocarpus y Ptelea, entre otros.

En las porciones montañosas se localiza el bosque de montaña, formado por: a) el bosque de encino, que se ubica en los cañones con mejores condiciones de humedad donde Quercus gravesii, $Q$. laceyi, $Q$. grisea y $Q$. saltillensis son las principales especies; b) el bosque de pino piñonero, dominado por Pinus cembroides o $P$. remota, es la comunidad boscosa más difundida en las zonas con clima templado semiseco; c) el bosque de pino, usualmente poco denso, integrado por Pinus rudis, $P$. arizonica var. stormiae y/o $P$. greggii, se presenta en las sierras donde se tiene clima templado semihúmedo. En elevaciones cercanas a los 3,000 m aparece: d) el bosque de oyamel, integrado principalmente por Pseudotsuga menziesii y e) el bosque de pino-encino, formado por una mezcla de especies del género Pinus, usualmente asociado con representantes de Quercus. En las cimas de los macizos montañosos de la Sierra de Arteaga, se localiza la vegetación alpina-subalpina, 
integrada por Pinus culminicola, Quercus greggii y Agave montana, además de una elevada riqueza de plantas herbáceas.

Los zacatales se desarrollan a través del estado creciendo en los valles con suelos profundos o en laderas bajas, con frecuencia se les encuentra mezclados con otros tipos de vegetación. La vegetación riparia, subacuática y acuática se restringe a los escasos cuerpos de agua permanentes, así como ríos y pozas, presentes en el centro y norte del área.

\section{MÉTODOS}

Con base en las colectas realizadas a través del área, además de la revisión bibliográfica y consulta de los herbarios ANSM, MEXU y TEX/LL (ver Holmgren et al. (1990) para los acrónimos), se definió un listado de la flora vascular para el estado de Coahuila. Se analizó la distribución geográfica de las especies con localidad tipo en la entidad y áreas vecinas, registrando aquellas restringidas a la región de estudio. Como parte del trabajo de recopilación de la información se elaboró una base de datos, en la cual además de la familia y del nombre de la especie se incluye información sobre el área de distribución y las condiciones ecológicas generales. Se prepararon cuadros de integración de datos, para finalmente indicar las regiones de mayor concentración de endemismo en Coahuila que pueden ser consideradas como zonas prioritarias de conservación.

\section{RESULTADOS}

La riqueza de plantas vasculares endémicas para Coahuila y algunas áreas adyacentes (Megacoahuila) consiste de 350 especies y taxa infraespecíficos (Apéndices 1 y 2), lo cual representa $11.2 \%$ del total de la flora nativa estimada para el estado. Son 190 las especies que limitan su distribución al territorio de la entidad administrativa, mismas que constituyen $54.5 \%$ de la cantidad total mencionada. Además 15 especies se enlistan en el Apéndice 2 como endémicas para Megacoahuila, pero no conocidas del estado.

El elemento endémico en la región está repartido en 50 familias, que representan 34\% del total. Las que incluyen 10 o más taxa de distribución restringida son: Asteraceae (75), Cactaceae (48), Lamiaceae (19), Fabaceae (17), Brassicaceae (15), Hydrophyllaceae (12), Agavaceae (12), Rosaceae -sensu lato- (11) y Acanthaceae (10). En el Cuadro 1 se presenta información sobre el total de taxa por grandes grupos taxonómicos. 
Cuadro 1. Participación cuantitativa de los grandes grupos de plantas vasculares en el conjunto de la flora de Coahuila y en el de sus especies endémicas (indicadas entre paréntesis). Se extiende esta información a los géneros y las familias a los que pertenecen tales elementos de distribución restringida.

\begin{tabular}{|l|c|c|c|}
\hline Grupo taxonómico & Familias & Géneros & $\begin{array}{c}\text { Especies y taxa } \\
\text { infraespecíficos }\end{array}$ \\
\hline Pteridophyta & $10(1)$ & $24(1)$ & $88(3)$ \\
\hline Pinophyta & $4(1)$ & $10(3)$ & $41(4)$ \\
\hline Magnoliophyta & $133(48)$ & $891(158)$ & $2,987(343)$ \\
\hline Magnoliopsida & $114(43)$ & $732(146)$ & $2,443(320)$ \\
\hline Liliopsida & $19(5)$ & $159(12)$ & $544(23)$ \\
\hline Total & $147(50)$ & $925(162)$ & $3,116(350)$ \\
\hline
\end{tabular}

Los géneros con representantes endémicos son 162, lo que constituye $17.5 \%$ del total. Tres son de distribución restringida a la región: Henricksonia, Marshalljohnstonia y Strotheria. Los de mayor número de taxa de repartición circunscrita se muestran en el Cuadro 2.

Cuadro 2. Géneros que presentan mayor número de taxa endémicos para Megacoahuila.

\begin{tabular}{|l|c|l|c|}
\hline \multicolumn{1}{|c|}{ Género } & Taxa & \multicolumn{1}{c|}{ Género } & Taxa \\
\hline Mammillaria & 10 & Brickellia & 7 \\
\hline Coryphantha & 9 & Opuntia & 7 \\
\hline Nama & 9 & Quercus & 7 \\
\hline Crataegus & 8 & Hedeoma & 6 \\
\hline
\end{tabular}


Entre las comarcas con el mayor número de endemismos destacan la de Cuatro Ciénegas, las Sierras del Carmen y Santa Rosa, de La Paila, de Parras y las de Jimulco y El Rosario, además de la Sierra Madre Oriental; otras regiones importantes aparecen en el Cuadro 3.

Los matorrales micrófilo y rosetófilo son los tipos de vegetación con el mayor número de endemismos, seguidos en importancia por el matorral submontano, el bosque de pino-encino y el matorral de halófitas. La representación correspondiente se presenta en el Cuadro 4.

\section{DISCUSIÓN}

El área de Coahuila (en su concepto de Megacoahuila) sobresale por su riqueza de endemismos regionales. Con casi $12 \%$ de su flora con distribución local, el área de estudio es un sitio importante para desarrollar acciones de conservación.

Cuadro 3. Áreas de Megacoahuila con mayor número de endemismos.

\begin{tabular}{|l|c|l|c|}
\hline \multicolumn{1}{|c|}{ Área } & Taxa & \multicolumn{1}{|c|}{ Área } & Taxa \\
\hline Cuatro Ciénegas & 58 & Sierra Las Delicias & 17 \\
\hline $\begin{array}{l}\text { Sierras del Carmen - } \\
\text { Santa Rosa }\end{array}$ & 46 & Sierra La Gavia & 16 \\
\hline Sierra de La Paila & 40 & Laguna del Rey & 12 \\
\hline Sierra de Parras & 33 & $\begin{array}{l}\text { Trans-Pecos y } \\
\text { Sierra del Pino }\end{array}$ & $11 \mathrm{c} / \mathrm{u}$ \\
\hline $\begin{array}{l}\text { Sierras de Jimulco - El } \\
\text { Rosario y Sierra Madre } \\
\text { Oriental }\end{array}$ & $30 \mathrm{c} / \mathrm{u}$ & $\begin{array}{l}\text { Hermanas y } \\
\text { Mapimí }\end{array}$ & 9 \\
\hline Sierra de la Madera & 24 & Viesca & \\
\hline
\end{tabular}


Cuadro 4. Número de taxa endémicos por tipo de vegetación.

\begin{tabular}{|l|c|l|c|}
\hline \multicolumn{1}{|c|}{ Tipos de vegetación } & Taxa & \multicolumn{1}{|c|}{ Tipos de vegetación } & Taxa \\
\hline $\begin{array}{l}\text { Matorrales micrófilo } \\
\text { y rosetófilo }\end{array}$ & 140 & Bosque de pino piñonero & 11 \\
\hline Matorral submontano & 82 & Zacatal & 7 \\
\hline $\begin{array}{l}\text { Bosque de pino encino } \\
\text { Matorrales de halófitas } \\
\text { gipsófitas }\end{array}$ & 58 & Bosque de oyamel & 3 \\
\hline Bosque de encino & 28 & Vegetación riparia & 2 \\
\hline Bosque de pino & 19 & Matorral tamaulipeco & 1 \\
\hline
\end{tabular}

Los elementos de distribución restringida son los que caracterizan una región y a la vez, representan las rarezas del área.

Los dos géneros con el mayor número de especies endémicas pertenecen a las cactáceas, lo que indica la importancia de la familia en este rubro y en cuanto a los requerimientos de protección.

La región de Cuatro Ciénegas sobresale por su flora endémica local y regional. Se compone en total de 58 especies que, sumadas a las de las sierras que bordean el valle, conforman una zona extremadamente rica en plantas de distribución restringida. Otros sectores importantes son las Sierras del Carmen y Santa Rosa en el norte del estado, con un número alto de endemismos (46). Ambas comarcas poseen programas de protección que requieren reforzarse en virtud de su riqueza florística. Áreas adicionales que destacan son las Sierras de La Paila (40), de Parras (33), la de Jimulco-El Rosario (30), y la Sierra Madre Oriental (30), que también merecen ser consideradas en proyectos de conservación. 
A pesar de que el estado de Coahuila se considera como bien explorado, es claro que todavía faltan datos sobre su flora, sobre todo de las sierras y valles alejados de la porción oeste del estado. La información que aquí se presenta estará sujeta a cambios a medida que se conozca mejor el universo vegetal de la región y la distribución de las especies.

Las plantas contempladas en la NOM-059-ECOL-2001 (lista de especies amenazadas, en peligro de extinción y sujetas a protección especial) con distribución en Coahuila (Anónimo, 2001), son principalmente cactáceas y agaváceas (Cuadro 5). Su mayor incidencia se presenta en Cuatro Ciénegas ( 8 especies), otras localidades sobresalientes son Sierra de La Paila (6), San Pedro (6), Viesca (6) y el sector ubicado entre Saltillo y Monterrey (4).

Las superficies naturales protegidas bajo la categoría de Áreas de Protección de Flora y Fauna de Sierra Maderas del Carmen y Cuatro Ciénegas (incluyendo las Sierras de La Madera y San Marcos) albergan en conjunto 108 taxa, lo que representa sólo $30.1 \%$ del total de las plantas endémicas. La Sierra de La Paila adiciona 21 taxa (6\%), la de Parras 7 (2\%), la de Jimulco $12(3.4 \%)$ y la Sierra Madre Oriental $10(2.9 \%)$. Si se desarrollaran programas tendientes a la conservación de estas zonas, se estaría protegiendo cerca de $44.4 \%$ de la flora endémica enlistada.

El presente listado, al ubicar las regiones con mayor número de endemismos, ofrece información de trascendencia para las autoridades competentes en la designación de reservas bióticas. Los datos proporcionados pueden servir para la formulación de listados oficiales de plantas bajo protección, programas de uso sustentable de especies vegetales o, en caso necesario, el rescate, la conservación y la propagación de entidades en peligro de extinción en jardines botánicos, como lo sugieren Vovides et al. (1997).

\section{AGRADECIMIENTOS}

Agradecemos las facilidades brindadas en la realización del presente trabajo a la Universidad Autónoma Agraria Antonio Narro. A Marco A. Llanas y Genaro Rodríguez por la elaboración del mapa de Megacoahuila, al Dr. Jorge S. Marroquín, del Departamento Forestal de la Universidad Autónoma Agraria Antonio Narro, por sus observaciones a una primera versión del manuscrito y al Dr. J. Rzedowski y los revisores por su aportación para mejorar su presentación. 
Cuadro 5. Especies de plantas endémicas de la región de estudio sujetas a protección oficial (NOM-059-ECOL-2001).

\begin{tabular}{|c|c|c|}
\hline Familia & Especie & Categoría de Conservación \\
\hline \multirow[t]{4}{*}{ Agavaceae } & Agave bracteosa & Amenazada \\
\hline & Agave parrasana & Sujeta a protección especial \\
\hline & Agave victoriae-reginae & En peligro de extinción \\
\hline & Yucca endlichiana & Sujeta a protección especial \\
\hline \multirow[t]{24}{*}{ Cactaceae } & Ariocarpus fissuratus & En peligro de extinción \\
\hline & Astrophytum capricorne & Amenazada \\
\hline & $\begin{array}{l}\text { Astrophytum myriostigma var. } \\
\text { coahuilense }\end{array}$ & Amenazada \\
\hline & Coryphantha durangensis & Sujeta a protección especial \\
\hline & Coryphantha pseudoechinus & Sujeta a protección especial \\
\hline & Coryphantha werdermanii & En peligro de extinción \\
\hline & Echinocereus longisetus & Sujeta a protección especial \\
\hline & Echinocereus nivosus & Sujeta a protección especial \\
\hline & Echinomastus mariposensis & Amenazada \\
\hline & Epithelantha bokei & Amenazada \\
\hline & Epithelantha micromeris var. greggii & Sujeta a protección especial \\
\hline & $\begin{array}{l}\text { Epithelantha micromeris var. } \\
\text { pachyrhiza }\end{array}$ & Sujeta a protección especial \\
\hline & Mammillaria carretii & Sujeta a protección especial \\
\hline & $\begin{array}{l}\text { Mammillaria coahuilensis var. } \\
\text { coahuilensis }\end{array}$ & Amenazada \\
\hline & Mammillaria grusonii & Sujeta a protección especial \\
\hline & Mammillaria guelzowiana & Amenazada \\
\hline & Mammillaria lenta & Amenazada \\
\hline & Mammillaria pennispinosa & Sujeta a protección especial \\
\hline & Mammillaria stella-de-tacubaya & Sujeta a protección especial \\
\hline & Opuntia anteojoensis & Sujeta a protección especial \\
\hline & Thelocactus bicolor var. bolaensis & Amenazada \\
\hline & Thelocactus macdowellii & Amenazada \\
\hline & Thelocactus rinconensis var. nidulans & Amenazada \\
\hline & Turbinicarpus mandragora & Amenazada \\
\hline Fouquieriaceae & Fouquieria shrevei & Sujeta a protección especial \\
\hline \multirow[t]{2}{*}{ Frankeniaceae } & Frankenia johnstonii & En peligro de extinción \\
\hline & Frankenia margaritae & Amenazada \\
\hline \multirow[t]{3}{*}{ Pinaceae } & Abies mexicana & Amenazada \\
\hline & Picea engelmanii var. mexicana & En peligro de extinción \\
\hline & Pinus culminicola & Sujeta a protección especial \\
\hline Rubiaceae & Galium carmenicola & Sujeta a protección especial \\
\hline
\end{tabular}




\section{LITERATURA CITADA}

Anónimo. 1983. Síntesis geográfica del estado de Coahuila, México. Instituto Nacional de Estadística Geografía e Informática. Secretaría de Programación y Presupuesto. México, D. F. 163 pp.

Anónimo. 2001. Especies y subespecies de flora y fauna silvestres terrestres y acuáticas en peligro de extinción, amenazadas, raras y las sujetas a protección especial. NOM059-ECOL. Secretaría de Medio Ambiente, Recursos Naturales y Pesca - Instituto Nacional de Ecología. Diario Oficial de la Federación. México D. F. 40 pp.

Elizondo, J. L., J. Valdés y A. Rodríguez. 1990. Cactáceas vulnerables y en peligro de extinción para Coahuila, México. Biotam 2(2): 17-22.

García, E. 1987. Modificaciones al sistema de clasificación climática de Köppen. 4a. ed. Universidad Nacional Autónoma de México. México, D.F. 217 pp.

Gómez-Pompa, A., R. Dirzo, A. Kaus, C. R. Noguerón-Chang y M. de J. Ordoñez. 1994. Reservas de la biosfera y otras áreas naturales protegidas de México. Secretaría del Medio Ambiente, Recursos Naturales y Pesca (SEMARNAP). México, D.F. 331 pp.

Henrickson, J. y M. C. Johnston. Manuscrito inédito. A flora of the Chihuahuan Desert region. $1687 \mathrm{pp}$.

Hernández, H. M. y H. Godínez A. 1994. Contribución al conocimiento de las cactáceas mexicanas amenazadas. Acta Bot. Mex. 26: 33-52.

Hinton, J. y G. Hinton. 1995. Checklist of Hinton's collections of the flora of south-central Nuevo Leon and adjacent Coahuila. Acta Bot. Mex. 30: 41-112.

Holmgren, P. K., N. H. Holmgren y L. C. Barnett (eds.). 1990. Index Herbariorum. Part I: The herbaria of the world. 8a. ed. The New York Botanical Garden. Nueva York. 693 pp.

Johnston, I. M. 1941. Gypsophily among Mexican desert plants. J. Arnold Arbor. 22(2): 145170.

McDonald, J. A. 1990. The alpine-subalpine flora of northeastern Mexico. Sida 14(1): 21-28.

Phipps, J. B. 1997. Monograph of northern Mexican Crataegus (Rosaceae, subfam. Maloideae). Sida, Botanical Miscellany 15:1-94.

Pinkava, D. J. 1984. Vegetation and flora of the Bolson of Cuatro Cienegas region, Coahuila, Mexico: IV Summary, endemism and corrected catalogue. J. Ariz.-Nev. Acad. Sci. 19: 23-47.

Rodríguez, A., J. Á. Villarreal y J. Valdés. 1994. Vegetación y flora de la Sierra de Parras, Coahuila. Agraria 10(2): 79-109.

Rzedowski, J. 1962. Contribuciones a la fitogeografía florística e histórica de México. I. Algunas consideraciones acerca del elemento endémico de la flora mexicana. Bol. Soc. Bot. Mex. 27: 52-65.

Rzedowski, J. 1991a. Diversidad y orígenes de la flora fanerogámica de México. Acta Bot. Mex. 14:3-21.

Rzedowski, J. 1991b. El endemismo en la flora fanerogámica mexicana: una apreciación analítica preliminar. Acta Bot. Mex. 15: 47-64. 
Vásquez, R., J. Á. Villarreal y J. Valdés. 1989. Las plantas de pastizales del rancho experimental ganadero Los Ángeles, Mpio. de Saltillo, Coah. México. Universidad Autónoma Agraria Antonio Narro. Folleto de divulgación 2(8): 1-20.

Villarreal, J. Á. 1994. Vegetación y flora de la Sierra de La Paila, Coahuila, México. Sida 16(1): 109-138.

Villarreal, J. Á. 2001. Flora de Coahuila. Listados florísticos de México. Instituto de Biología. Universidad Nacional Autónoma de México. México D.F. 136 pp.

Villarreal, J. Á. y J. Valdés. 1992-1993. Vegetación de Coahuila, México. Revista de Manejo de Pastizales 6(1,2): 9-18.

Villarreal, J. Á., J. Valdés y J. L. Villaseñor. 1996. Corología de las asteráceas de Coahuila, México. Acta Bot. Mex. 36: 29-42.

Villaseñor, J. L. 1991. Las Heliantheae endémicas a México: una guía hacia la conservación. Acta Bot. Mex. 15: 29-46.

Villaseñor, J. L., G. Ibarra y D. Ocaña. 1998. Strategies for the conservation of Asteraceae in Mexico. Conservation Biology 12(5): 1066-1075.

Vovides, A. P., V. Luna y G. Medina. 1997. Relación de algunas plantas y hongos raros, amenazados o en peligro de extinción y sugerencias para su conservación. Acta Bot. Mex. 39: 1-42. 
Apéndice 1. Listado de los taxa endémicos a Coahuila y algunas áreas adyacentes.

\section{PTERIDOPHYTA}

Selaginellaceae

Selaginella carnerosana Reeves. Matorral submontano y bosque de pino piñonero, laderas rocosas. Sierra de Carneros en Coah.

Selaginella parishii Underw. Matorral submontano, laderas rocosas. S de Coah. (Sierra de Parras) y $\mathrm{N}$ de Zac. (municipio de Concepción del Oro).

Selaginella viridissima Weath. Matorrales submontano, micrófilo y rosetófilo, laderas rocosas. Sierras Mojada, La Fragua, y de La Gloria en Coah. y las Montañas Chisos en TX.

\section{PINOPHYTA (GYMNOSPERMAE)}

Pinaceae

Abies durangensis Martínez var. coahuilensis (I. M. Johnst.) Martínez (A. coahuilensis I. M. Johnst.). Bosque de oyamel con suelo rocoso, cimas de las sierras. Sierras de La Madera, de La Gloria, del Carmen, de Santa Rosa y Serranías del Burro en Coah.

Abies mexicana Martínez. Bosques de oyamel en laderas y cañones. Sierra Madre Oriental, Arteaga en Coah. y áreas adyacentes de N.L.

Picea engelmannii Parry var. mexicana (Martínez) Silba (P. mexicana Martínez). Bosque de oyamel en cañones, laderas rocosas. Sierra Madre Oriental, Sierra de La Marta, Sierra de Arteaga en Coah. y municipio de Rayones en N.L.

Pinus culminicola Andresen \& Beaman. Bosque de pino-encino en cimas montañosas entre 3000 a 3600 m. Sierra Madre Oriental, sierras de La Marta y La Viga en Coah. y parte de Sierra de La Marta, así como el cerro El Potosí en N.L.

\section{MAGNOLIOPSIDA (DICOTYLEDONEAE)}

\section{Acanthaceae}

Anisacanthus junceus (Torr.) Hemsl. Matorrales micrófilo, rosetófilo y submontano, laderas bajas y arroyos. Sierras de Jimulco y de Parras, así como el municipio de Viesca en Coah. y $\mathrm{N}$ de Zac. 
Apéndice 1. Continuación.

Carlowrightia mexicana Henr. \& T. F. Daniel. Matorral submontano, valles y arroyos pedregosos. N y centro de Coah. (Cuatro Ciénegas, Sierra de La Gloria), NE de Chih. y región de Trans-Pecos, TX.

Carlowrightia parvifolia Brandegee. Matorrales micrófilo y rosetófilo, laderas rocosas y arroyos. NE y SE de Coah. (sierras de La Paila y de La Madera), NE de Chih. y región de Trans-Pecos, TX.

Carlowrightia serpyllifolia A. Gray. Matorrales micrófilo y rosetófilo, valles y laderas rocosas. N y centro de Coah. (Valle de Cuatro Ciénegas, Sierra de La Paila), NE de Chih., NE de Dgo., N de Zac. y región de Trans-Pecos, TX.

Holographis ilicifolia Brandegee. Matorrales micrófilo y rosetófilo, laderas rocosas, valles y arroyos pedregosos. Valle de Cuatro Ciénegas, así como Sierra de Jimulco en Coah. y Sierra de El Rosario en Dgo.

Justicia coahuilana T. F. Daniel. Matorral submontano, laderas rocosas. Sierra de La Madera en Coah.

Justicia decurvata Hilsenb. (Siphonoglossa lineriafolia Henr. \& Hilsenb.). Matorrales micrófilo y rosetófilo, partes bajas de las sierras. Sierra de Jimulco en Coah., NE de Dgo. (Río Nazas).

Justicia henricksonii T. F. Daniel. Matorral submontano, laderas rocosas. W y centro de Coah. (Sierra del Pino) y N de S.L.P.

Justicia warnockii T. F. Daniel. Matorrales micrófilo y rosetófilo, laderas bajas. W de Coah., E de Chih., región de Trans-Pecos, TX.

Ruellia jimulcensis Villarreal. Matorrales micrófilo y rosetófilo, laderas rocosas y valles. Sierra de Jimulco en Coah. y Sierra de El Rosario en Dgo.

Amaranthaceae

Tidestromia gemmata I. M. Johnst. Matorral de halófitas, suelos yesosos. Valle de Cuatro Ciénegas en Coah., NE de Chih. y región de Trans-Pecos, TX.

Tidestromia rhizomatosa I. M. Johnst. Matorral de halófitas, suelos arenosos y salinos. Valle de Cuatro Ciénegas en Coah.

Tidestromia suffruticosa (Torr.) Standl. var. coahuilana I. M. Johnst. Matorrales micrófilo y rosetófilo, laderas bajas con suelos pedregosos. Sierras de Las Delicias, de La Paila y del Pino en Coah. 
Apéndice 1. Continuación.

Tidestromia tenella I. M. Johnst. Matorrales micrófilo y rosetófilo, valles con suelos arenosos. Valles de Cuatro Ciénegas, Viesca y Mohovano en Coah.

Apiaceae (Umbelliferae)

Aletes calcicola Mathias \& Constance. Bosque de pino-encino y matorral submontano, suelo rocoso. Sierras de La Gloria, de San Marcos, de La Madera y de La Gavia en Coah.

Aletes coahuilensis Mathias \& Constance. Bosque de pino-encino en laderas rocosas. Sierras del Carmen, Santa Rosa y del Pino en Coah.

Donnellsmithia coahuilensis Mathias \& Constance. Bosque de pino-encino, suelo rocoso. Sierra del Pino en Coah.

Tauschia hintoniorum Constance \& Affolter. Bosque de pino-encino en laderas rocosas. Sierra Madre Oriental, Sierra de La Marta en Coah. y áreas adyacentes con N.L.

Aristolochiaceae

Aristolochia lassa I. M. Johnst. Matorrales micrófilo y rosetófilo en suelo pedregoso. S de Coah. y SW de N.L.

Asteraceae (Compositae)

Ageratina astellera (B. L. Turner) B. L. Turner (Eupatorium astellerum B. L. Turner). Matorral submontano en arroyos con suelo pedregoso. S de Coah. y N de Zac. (Sierra del Astillero).

Ageratina campyloclada (B. L. Rob.) R. M. King \& H. Rob. (Eupatorium campylocladum B. L. Rob.). Matorral submontano y bosque de coníferas en laderas rocosas. Sierra Madre Oriental, SE de Coah. y NW de N.L.

Ageratina flourensifolia (B. L. Turner) R. M. King \& H. Rob. (Eupatorium flourensifolium B. L. Turner). Bosque de pino-encino y matorral submontano en laderas rocosas. Sierras de San Marcos y de La Gloria en Coah.

Ageratina glischra (B. L. Rob.) R. M. King \& H. Rob. (Eupatorium glischrum B. L. Rob.) Matorral submontano y bosque de pino-encino en laderas rocosas. Sierras de Jimulco, de Parras y de La Madera en Coah.

Ageratina riskindii B. L. Turner. Bosque de encino, en laderas. Sierra de Santa Rosa (Rincón de María) en Coah. 
Apéndice 1. Continuación.

Ageratina zapalinama B. L. Turner. Bosque de pino-encino, laderas rocosas. Sierra Madre Oriental, municipios de Saltillo y Arteaga en Coah. y áreas colindantes de N.L.

Ambrosia johnstoniorum Henr. Zacatal en valles con suelo arcilloso. Sierras de La Madera y Cruces (Cuesta de Zozoya) en Coah.

Brickellia glutinosa A. Gray. Matorral micrófilo y rosetófilo en lomeríos con suelo pedregoso. Valle de Cuatro Ciénegas, Sierra Mojada y Sierra de Jimulco en Coah., posiblemente áreas adyacentes de Chih.

Brickellia hintoniorum B. L. Turner. Bosque de coníferas, en laderas rocosas. Sierra Madre Oriental, municipio de Arteaga (sierras del Coahuilón, de La Marta y de La Viga) en Coah. y áreas colindantes de N.L.

Brickellia laccata Flyr. Matorral submontano, en laderas rocosas. Sierra del Carmen en Coah.

Brickellia lemmonii A. Gray. var. carmenensis B. L. Turner. Bosque de encino, en laderas rocosas. Sierra del Carmen en Coah.

Brickellia stolonifera B. L. Turner. Bosque de coníferas, en laderas. Sierra del Carmen en Coah.

Brickellia urolepis Blake. Bosque de encino y pino-encino, en laderas. Sierras del Carmen, de La Gavia, de La Paila y de La Madera en Coah.

Brickellia wendtii B. L. Turner. Bosque de encino, en laderas. Sierra de Santa Rosa (Rincón de María) en Coah.

Chaetopappa plomoensis B. L. Turner. Matorrales micrófilo y rosetófilo, en laderas rocosas. Sierras del Carmen y Santa Rosa en Coah.

Chaetoppapa pulchella Shinners. Matorral submontano, en laderas pedregosas. Valle de Cuatro Ciénegas y Sierra Mojada en Coah. y E de Chih.

Cirsium coahuilense Ownbey \& Pinkava. Matorrales micrófilo y rosetófilo en valles, lomeríos y lugares inundados. Montañas bajas y represas del S de Coah.

Cirsium turneri Warnock. Matorral submontano y bosque de encino, en laderas rocosas. NW de Coah. (Sierra del Carmen) en Coah., montañas del NE de Chih. y áreas adyacentes con TX. 
Apéndice 1. Continuación.

Erigeron chiangii G. L. Nesom. Matorral submontano y bosque de encino, en laderas rocosas. E y centro de Coah. (Valle de Cuatro Ciénegas).

Erigeron cuatrocienegensis G. L. Nesom. Matorrales micrófilo y rosetófilo en valles con suelos yesosos. Valle de Cuatro Ciénegas en Coah.

Erigeron hintoniorum B. L. Turner. Bosque de pino-encino en laderas rocosas. Sierra Madre Oriental, municipio de Arteaga en Coah. y áreas colindantes de N.L.

Erigeron pinkavii B. L. Turner. Matorral de halófitas en valles con suelos salinos. Valle de Hermanas al $\mathrm{N}$ de Monclova en Coah. y áreas adyacentes de N.L.

Flaveria palmeri J. R. Johnst. Matorral de halófitas en suelos salinos y yesosos. Municipios de Cuatro Ciénegas, San Pedro, Matamoros y Parras, así como Laguna del Rey en Coah. y cerca de Monterrey en N.L.

Flourensia ilicifolia Brandegee. Matorrales micrófilo y rosetófilo en valles y laderas con suelo pedregoso. Sierra de Parras en Coah.

Flourensia microphylla (A. Gray) Blake. Matorral submontano en laderas pedregosas. Sierras de Carneros y de San Marcos, en el municipio de Saltillo en Coah.

Flourensia monticola M. O. Dillon. Bosque de pino-encino, laderas y cañones. Sierra Madre Oriental, municipios de Arteaga en Coah., así como de Santa Catarina y Santiago en N.L.

Flourensia pulcherrima M. O. Dillon. Matorral submontano, laderas rocosas. Sierras de Jimulco en Coah. y El Rosario en Dgo. y SE de Chih.

Flourensia retinophylla Blake. Matorral submontano en cañones y suelos pedregosos. Sierras de Las Delicias, de La Madera, de La Paila y Bolsón de Mapimí en Coah.

Flourensia solitaria Blake. Matorrales micrófilo y rosetófilo, suelo pedregoso. Valle de Cuatro Ciénegas (Cuesta Zozaya) en Coah.

Gaillardia gypsophila B. L. Turner. Matorral de halófitas, en dunas yesosas. Valle de Cuatro Ciénegas en Coah.

Gaillardia henricksonii B. L. Turner. Matorral de halófitas en valles con suelo yesoso. Municipio de Ocampo en Coah.

Gaillardia powellii B. L. Turner. Matorral de halófitas en valles con suelo yesoso. Valle de Hermanas al $\mathrm{N}$ de Monclova en Coah. y áreas adyacentes de N.L. 
Apéndice 1. Continuación.

Haploesthes fruticosa B. L. Turner. Matorrales micrófilo y rosetófilo, en laderas con suelos pedregosos. Sierra de La Paila en Coah.

Haploesthes robusta I. M. Johnst. Matorral de halófitas en valles con suelo yesoso. Valle de Cuatro Ciénegas en Coah.

Heliopsis filifolia S. Watson. Matorrales micrófilo y rosetófilo en suelos pedregosos y arenosos. Sierras de Parras, de La Fragua y de Carneros en Coah.

Henricksonia mexicana B. L. Turner. Matorrales micrófilo y rosetófilo en laderas rocosas. Sierra de Jimulco en Coah. y NE de Dgo. (Río Nazas).

Hymenopappus hintoniorum B. L. Turner. Bosque de pino-encino, laderas rocosas. Sierra Madre Oriental, municipio de Arteaga (Sierra del Coahuilón) en Coah. y áreas adyacentes del $\mathrm{W}$ de N.L.

Hymenoxys insignis (A. Gray) Cockerell (H. ursina Standl.). Bosque de pino-encino y coníferas, suelo rocoso. Sierra Madre Oriental, Sierras de La Marta, del Coahuilón y de la Viga en Coah. y cerro El Potosí en N.L.

Machaeranthera johnstonii (Blake) B. L. Turner. Matorral de halófitas en suelo yesoso. Valle de Hermanas al $\mathrm{N}$ de Monclova en Coah. y áreas adyacentes con N.L.

Machaeranthera restiformis B. L. Turner. Matorral de halófitas en dunas yesosas. Valle de Cuatro Ciénegas en Coah.

Marshalljohnstonia gypsophila Henr. Matorral submontano en laderas yesosas. Sierra de Las Delicias en Coah.

Melampodium argophyllum (B. L. Rob.) Blake (M. cinereum DC. var. argophyllum B. L. Rob.). Matorrales micrófilo, rosetófilo y submontano, suelo pedregoso. Centro y S de Coah. (municipios de G. Cepeda y Parras, sierras de La Gavia y de San Marcos) y W de N.L. (Icamole).

Pectis incisifolia I. M. Johnst. Matorrales micrófilo y rosetófilo en valles con suelos salinos. Bolsón de Mapimí (área limitrofe entre Coah., Chih. y Dgo.).

Perityle bisetosa (A. Gray) Shinners var. spathulata Powell. Matorral submontano en laderas rocosas. Sierra del Carmen en Coah.

Perityle carmenensis Powell. Matorrales microfilo y rosetófilo, en laderas rocosas. Sierra del Carmen en Coah. 
Apéndice 1. Continuación.

Perityle castillonii I. M. Johnst. Matorral submontano en cañones y laderas rocosas. Sierra Hechiceros (W de Coah. y SE de Chih.).

Perityle coahuilensis Powell. Matorral submontano y bosque de encino en laderas rocosas. Sierra de La Madera (El Sobaco) en Coah.

Pinaroppapus mojadanus B. L. Turner. Matorral submontano en laderas rocosas. Sierra Mojada en Coah.

Pinaroppapus powellii B. L. Turner. Bosque de pino-encino en laderas rocosas. Sierra La Encantada en Coah.

Porophyllum amplexicaule A. Gray. Matorrales micrófilo y rosetófilo en laderas pedregosas. Cerca de Saltillo en Coah. y áreas colindantes con N.L.

Psathyrotopsis hintoniorum B. L. Turner. Matorrales micrófilo y rosetófilo en laderas bajas con suelos pedregosos. Sierra de Parras en Coah.

Psathyrotopsis purpusii (Brandegee) Rydb. (Psathyrotes purpusii Brandegee). Matorrales micrófilo y rosetófilo en laderas bajas con suelo arenoso. Marte, al S de la Sierra de La Paila en Coah.

Ratibida coahuilensis B. L. Turner. Matorral submontano y bosque de encino en suelos pedregosos. Sierra de Santa Rosa en Coah. y W de N.L.

Sartwellia mexicana A. Gray. Matorral de halófitas, en valles con suelo arcilloso y yesoso. Centro de Coah. (Valle de Cuatro Ciénegas), W de N.L., N de S.L.P. y NE de Zac.

Senecio claryae B. L. Turner. Matorral submontano y bosque de encino en suelos rocosos. Sierra de La Paila en Coah.

Senecio riskindii B. L. Turner \& T. M. Barkley. Bosque de coníferas en suelo rocoso. Sierra del Carmen en Coah.

Senecio scalaris Greene var. carmenensis C. C. Freeman. Bosque de pino-encino, suelo pedregoso. Sierra del Carmen en Coah.

Senecio scalaris Greene var. parrasianus (Greenm.) C. C. Freeman. Bosque de pino-encino, suelo pedregoso. Sierra de Parras en Coah.

Solidago gypsophila G. L. Nesom. Matorrales micrófilo y rosetófilo, cerca de corrientes de agua en suelos yesosos. Valle de Cuatro Ciénegas y Sierra de San Marcos en Coah. 
Apéndice 1. Continuación.

Thelesperma scabridulum Blake. Matorral de halófitas en valles con suelo yesoso. Áreas salinas del SE de Coah. y NW de N.L.

Thelesperma subaequale Blake. Bosque de encino en laderas y cañones. Sierras del Carmen, del Pino, de Santa Rosa en Coah. y centro de N.L.

Thymophylla gypsophila (B. L. Turner) Strother. Matorral de halófitas con suelo yesoso. Valle de Cuatro Ciénegas y Laguna del Rey en Coah.

Varilla mexicana A. Gray var. gypsophila B. L. Turner. Matorrales micrófilo y rosetófilo, en suelos yesosos. Valle de Cuatro Ciénegas en Coah.

Varilla mexicana A. Gray var. mexicana. Matorrales micrófilo y rosetófilo, en escurrimientos. $\mathrm{S}$ de Coah., NE de Dgo. y $\mathrm{N}$ de Zac.

Verbesina coahuilensis A. Gray var. viridior B. L. Rob. \& Greenm. Bosque de pino-encino, laderas pedregosas. Sierra Madre Oriental, S de Coah. (sierras de Carneros, Zapalinamé y de Arteaga) y áreas adyacentes de N.L.

Verbesina daviesiae B. L. Turner. Bosque de pino-encino, laderas rocosas. Sierra Madre Oriental, Sierra de El Coahuilón y Sierra de La Viga en Coah. y posiblemente áreas adyacentes de N.L.

Vernonia faustiana (G. C. Chapm. \& S. B. Jones) B. L. Turner. Bosque de encino, en laderas. Sierra Santa Rosa (Rincón de María) en Coah.

Viguiera brevifolia Greenm. Matorrales micrófilo y rosetófilo en valles, lomeríos y arroyos. Sierra de Jimulco en Coah., NE de Dgo. y SE de Chih.

Viguiera phenax Blake. Zacatal y matorrales micrófilo y rosetófilo, en valles con suelo arcilloso. W de Coah., NE de Dgo., E de Chih. y región de Trans-Pecos, TX.

Xylothamia parrasana (Blake) G. L. Nesom (Ericameria parrasana Blake). Matorral submontano en laderas rocosas. Sierra de Parras en Coah. y N de Zac.

Xylothamia pseudobaccharis (Blake) G. L. Nesom (Haplopappus pseudobaccharis Blake) Matorrales micrófilo y rosetófilo, en suelos pedregosos y yesosos. Sierras de Las Delicias, de los Órganos, de La Paila y Alamitos en Coah. y NE de Dgo.

Xylothamia riskindii (B. L. Turner \& G. Langford) G. L. Nesom (Ericameria riskindii B. L. Turner \& G. Langford). Bosque de encino en laderas rocosas. Sierra Madre Oriental, Sierra de Arteaga en Coah. y áreas adyacentes con N.L. 
Apéndice 1. Continuación.

Xylothamia truncata G. L. Nesom. Matorral de halófitas en suelos salinos y yesosos. Valle de Cuatro Ciénegas en Coah.

Zaluzania megacephala Sch. Bip. var. coahuilensis Olsen. Bosque de encino en laderas rocosas. SE de Coah. (municipio de General Cepeda) y E de N.L.

Zinnia oligantha I. M. Johnst. Matorral de halófitas en valles con suelos yesosos y dunas. Laguna del Rey en Coah. y E de Chih.

Berberidaceae

Berberis pinifolia (Lundell) C. H. Müll var. coahuilensis C. H. Müll. Matorral submontano, laderas pedregosas. Sierra de La Madera en Coah.

Boraginaceae

Lithospermum jimulcense I. M. Johnst. Matorral submontano y bosque de pino piñonero, suelo rocoso. Sierra de Jimulco en Coah.

Omphalodes chiangii Higgins. Bosque de pino-encino, suelos arenosos. Sierras del Carmen y Santa Rosa en Coah.

Tiquilia mexicana (S. Watson) A. Richardson. Matorral micrófilo y rosetófilo, en valles y laderas. Coah., W de N.L., N de Zac., E de Chih. y región de Trans-Pecos, TX.

Tiquilia turneri A. Richardson. Matorral de halófitas en suelos yesosos. Valle de Cuatro Ciénegas en Coah. y W de N.L.

Brassicaceae (Cruciferae)

Lesquerella inflata Rollins \& E. A. Shaw. Bosque de pino piñonero, suelo rocoso. Sierra Madre Oriental, Sierra de los Nuncios en Coah. y áreas aledañas de N.L.

Lesquerella johnstonii Rollins. Bosque de pino-encino, suelo pedregoso y arroyos. Sierras del Carmen, de Santa Rosa y del Pino en Coah.

Lesquerella mcvaughiana Rollins. Matorral submontano, laderas pedregosas. Coah. (Valle de Cuatro Ciénegas), Pecos y Brewster Co., TX.

Lesquerella mexicana Rollins. Matorral submontano, suelo pedregoso. Valle de Cuatro Ciénegas, sierras de Menchaca, de Parras, del Carmen, de Santa Rosa y La Encantada en Coah. y E de Chih. 
Apéndice 1. Continuación.

Lesquerella wyndii Rollins \& E. A. Shaw. Bosque de pino-encino, en arroyos. Sierras del Carmen y Santa Rosa en Coah.

Nerisyrenia baconiana B. L. Turner. Bosque de pino-encino en suelo yesoso. Sierra de La Paila en Coah.

Nerisyrenia castillonii Rollins (N. powellii J. D. Bacon). Matorral de halófitas, suelo yesoso. Laguna del Rey en Coah. y NE de Chih.

Nerisyrenia incana Rollins. Matorral de halófitas en suelo yesoso. Valle de Cuatro Ciénegas en Coah.

Nerisyrenia johnstonii J. D. Bacon. Matorral de halófitas en suelo yesoso. Sierra de Las Delicias y SW de Coah.

Sphaerocardamum compressum (Rollins) Rollins. Matorral submontano en suelo yesoso. Sierra de Parras en Coah.

Streptanthus coulteri Cory. Matorral submontano, suelo pedregoso. Sierras del Carmen y Santa Rosa en Coah., E de Chih. y región de Big Bend en TX.

Streptanthus platycarpus A. Gray. Matorral submontano, laderas rocosas. Sierras del Carmen y Santa Rosa en Coah., W de TX.

Synthlipsis densiflora Rollins. Bosque de pino-encino, laderas rocosas. Valle de Cuatro Ciénegas y Sierra de La Fragua en Coah.

Thelypodiopsis incisa Rollins. Matorral submontano, laderas rocosas. Sierras de La Gavia, de San Marcos y municipio de Saltillo en Coah.

Thelypodiopsis retrofracta (Rollins) Rollins (Sisymbrium retrofractum Rollins). Matorrales micrófilo y rosetófilo, suelo rocoso. Sierra de Parras en Coah. y N de Zac.

Buddlejaceae

Emorya rinconensis Mayfield. Matorral submontano, laderas de cañones. Serranías del Burro en Coah.

Emorya suaveolens Torr. Matorral submontano y bosque de encino, laderas y arroyos. Sierras de La Madera, de Parras, de La Fragua, de La Gavia y de La Paila en Coah., W de N.L. y región de Trans-Pecos, TX. 
Apéndice 1. Continuación.

\section{Cactaceae}

Ariocarpus fissuratus (Engelm.) K. Schum. var. lloydii (Rose) W. T. Marshall. Matorrales micrófilo y rosetófilo, lomeríos y partes bajas de las sierras. Sierras de Parras y de La Paila en Coah., $\mathrm{N}$ de Zac. y NE de Dgo.

Astrophytum capricorne (A. Dietr.) Britton \& Rose. Matorrales micrófilo y rosetófilo, suelos pedregosos en faldas de sierras. Municipios de Cuatro Ciénegas y San Pedro, S de Coah. y W de N.L.

Astrophytum myriostigma Lem. var. coahuilense (H. Moeller) Borg. Matorrales micrófilo y rosetófilo, suelo pedregoso. Municipio de San Pedro en Coah. y NE de Dgo.

Coryphantha borwigii Purpus. Matorrales micrófilo y rosetófilo en laderas rocosas y lomeríos. Centro y SE de Coah. y W de N.L. (corredor Saltillo-Monterrey).

Coryphantha difficilis (Quehl) Orcutt (Mammillaria difficilis Quehl). Matorrales micróflio y rosetófilo, laderas de sierras. Sierra de La Paila en Coah.

Coryphantha durangensis (Runge ex K. Schum.) Britton \& Rose (Mammillaria durangensis Runge ex K. Schum.). Matorrales micrófilo y rosetófilo, afloramientos rocosos. SW de Coah. (municipios de Viesca y San Pedro) y NE de Dgo. (Río Nazas).

Coryphantha laredoi Glass \& Foster (Escobaria laredoi (Glass \& Foster) N. P. Taylor). Matorrales micrófilo y rosetófilo, laderas rocosas. Sierra de Parras en Coah. y N de Zac.

Coryphantha lloydii (Britton \& Rose) Fosberg (Escobaria lloydii Britton \& Rose). Matorrales micrófilo y rosetófilo, suelos pedregosos. S de Coah. y N de Zac.

Coryphantha pseudoechinus Boed. Matorrales micrófilo y rosetófilo, laderas rocosas. Centro y S de Coah. (Valle de Cuatro Ciéneagas, Sierras de San Marcos y de La Paila).

Coryphantha unicornis Boed. Matorrales micrófilo y rosetófilo, lomeríos. SW de Coah. (municipio de Viesca).

Coryphantha werdermannii Boed. (C. densispina Werderm.). Matorrales micrófilo y rosetófilo, en valles y laderas bajas. Sierra de La Paila en Coah. y E de Chih. (Sierra El Diablo).

Coryphantha zilziana Boed. (Escobaria zilziana (Boed.) Backeb.). Matorrales micrófilo y rosetófilo, afloramientos rocosos. Centro y SW de Coah. (Valle de Cuatro Ciénegas), NE de Dgo. (Río Nazas) y W de N.L. 
Apéndice 1. Continuación.

Echinocereus chisoensis W. T. Marshal. var. fobeanus (Oehme) N. P. Taylor (E. fobeanus Oehme). Matorrales micrófilo y rosetófilo. Valles y partes altas de sierras. SW de Coah.

Echinocereus longisetus (Engelm.) Lem. var. delaetii (M. Guerke) N. P. Taylor (E. delaetii M. Guerke). Zacatal, bosque de encino y bosque de pino piñonero. Sierras de La Paila y de San Marcos en Coah.

Echinocereus longisetus (Engelm.) Lem. var. longisetus. Bosque de pino-encino, laderas rocosas. Sierras de La Madera y del Carmen en Coah.

Echinocereus nivosus Glass \& Foster. Zacatal y bosque de pino piñonero, en laderas y valles. SE de Coah. (cerca Sierra de Carneros).

Echinocereus primolanatus N. P. Taylor. Matorrales micrófilo y rosetófilo, laderas expuestas de sierras. Sierras de La Paila y de Los Alamitos en Coah.

Echinomastus mariposensis Hester. Matorrales micrófilo y rosetófilo y zacatal, en valles y laderas rocosas. Valle de Cuatro Ciénegas y S de Coah., alrededores de Presidio cerca del Río Bravo en Brewster Co., TX.

Epithelantha bokei L. D. Benson (E. micromeres (Engelm.) Britton \& Rose var. bokei (L. D. Benson) Glass \& Foster). Matorrales micrófilo, rosetófilo y submontano, en afloramientos rocosos. Valle de Cuatro Ciénegas y Sierra de La Paila en Coah. y cerca de Río Grande, TX.

Epithelantha micromeris (Engelm.) Britton \& Rose var. greggii (Engelm.) Borg. Matorrales micrófilo y rosetófilo, en afloramientos rocosos. S de Coah.

Epithelantha micromeris (Engelm.) Britton \& Rose var. pachyrhiza W. T. Marshall Matorral submontano, laderas rocosas. E de Coah. y W de N.L.

Epithelantha micromeris (Engelm.) Britton \& Rose var. polycephala (Backeb.) Glass \& Foster. Matorrales micrófilo y rosetófilo, lomeríos. SE de Coah.

Grusonia bradtiana (Coult.) Ferguson (Opuntia bradtiana (Coult.) K. Brandegee). Matorrales micrófilo y rosetófilo, valles, lomeríos y partes bajas de sierras. Centro y $\mathrm{S}$ de Coah., SE de Chih. y NE de Dgo.

Gymnocactus aguirreanus Glass \& Foster (Escobaria aguirreana (Glass \& Foster) N. P. Taylor). Matorrales micrófilo y rosetófilo, laderas. Sierras de La Paila y de San Marcos en Coah. 
Apéndice 1. Continuación.

Mammillaria albiarmata Boed. Matorrales micrófilo y rosetófilo, valles. SE de Coah. (municipio de Saltillo).

Mammillaria carretii K. Schum. Matorrales micrófilo y rosetófilo, laderas rocosas. E de Coah. y W de N.L. (corredor Saltillo-Monterrey).

Mammillaria coahuilensis (Boed.) Moran var. coahuilensis. Matorral de halófitas, valles inundables. Laguna de Viesca en Coah.

Mammillaria freudenbergeri Repp. Matorrales micrófilo y rosetófilo, valles. Centro de Coah. (municipio de Monclova).

Mammillaria grusonii Runge. Matorrales micrófilo y rosetófilo, laderas rocosas. Municipios de Cuatro Ciénegas y San Pedro en Coah. y NE de Dgo.

Mammillaria lenta $\mathrm{K}$. Brandegee. Matorrales micrófilo y rosetófilo, lomeríos. Centro y SW de Coah. (municipio de Viesca) y cerca de Monterrey N.L.

Mammillaria parrasensis Repp. Matorrales micrófilo y rosetófilo, en afloramientos rocosos. Sierra de Parras en Coah. y N de Zac.

Mammillaria pennispinosa Krainz var. pennispinosa. Matorrales micrófilo y rosetófilo, laderas rocosas. Bolsón de Mapimí en los límites de Coah., Dgo. y Chih.

Mammillaria ritteriana Boed. Matorrales micrófilo y rosetófilo, en laderas rocosas. E de Coah. y W de N.L. (corredor Saltillo-Monterrey).

Mammillaria stella-de-tacubaya Hesse (M. tacubayensis Fedde). Matorrales micrófilo y rosetófilo, en laderas rocosas. SW de Coah. (bolsones de Viesca y Mapimí) y NE de Dgo. (Río Nazas).

Opuntia aggeria Ralston \& Hilsenb. Matorrales micrófilo y rosetófilo, suelos arenosos en valles y partes bajas de sierras. Centro de Coah. y Brewster Co., TX.

Opuntia anteojoensis Pinkava. Matorral de halófitas, valles con suelos yesosos. Valle de Cuatro Ciénegas en Coah.

Opuntia aureispina (Brack. \& Heil) Pinkava \& Parfitt. Matorrales micrófilo y rosetófilo, valles. N de Coah. región del Río Bravo, de Big Bend y Brewster Co., TX.

Opuntia bulbispina Engelm. Matorrales micrófilo y rosetófilo, suelos rocosos en partes bajas de sierras. Abanicos aluviales y Valles de Coahuila. 
Apéndice 1. Continuación.

Opuntia chisosensis (Anthony) Ferguson (O. lindheimeri Engelm. var. chisosensis Anthony). Zacatal y bosques de pino piñonero y pino-encino. Sierra del Carmen y Montañas Chisos, TX.

Opuntia moelleri Berger. Matorrales micrófilo y rosetófilo con suelos yesosos, arenosos y rocosos en valles y partes bajas de sierras. Valle de Cuatro Ciénegas en Coah.

Opuntia pailana Weing. Matorrales micrófilo y rosetófilo, en partes bajas de la sierra con suelos rocosos. Sierra de La Paila en Coah.

Thelocactus bicolor (Pfeiff.) Britton \& Rose var. bolaensis (Runge) A. Berger. Matorrales micrófilo y rosetófilo, lomeríos con afloramientos rocosos. Entre Paila y San Pedro en Coah.

Thelocactus lausseri Riha \& Busek. Matorrales micrófilo y rosetófilo, en partes bajas de la Sierra Las Ovejas (centro de Coah.).

Thelocactus macdowellii (Quehl) Glass. Matorrales micrófilo y rosetófilo, en lomeríos. E de Coah. y W de N.L. (corredor Saltillo-Monterrey).

Thelocactus rinconensis (Poselg.) Britton \& Rose var. nidulans (Quehl) Glass \& Foster (T. nidulans (Quehl) Britton \& Rose). Matorrales micrófilo y rosetófilo, en lomeríos. Municipios de San Pedro, Saltillo en Coah. y W de N.L. (Icamole-Monterrey).

Thelocactus rinconensis (Poselg.) Britton \& Rose var. phymatothelos (Ruempler) Glass \& Foster (T. phymatothelos (Ruempler) Britton \& Rose). Matorrales micrófilo y rosetófilo, suelo pedregoso. SE de Coah.

Thelocactus rinconensis (Poselg.) Britton \& Rose var. rinconensis. Matorrales micrófilo y rosetófilo, lomeríos. SE de Coah. y W de N.L.

Turbinicarpus mandragora (A. Berger) A. D. Zimmerman. Matorrales micrófilo y rosetófilo, laderas rocosas. Sierra de Parras y municipio de Viesca en Coah.

Campanulaceae

Lobelia anatina Wimm. var. riskindii M. C. Johnst. Bosque de coníferas, arroyos. Sierra del Carmen en Coah.

Lobelia henricksonii M. C. Johnst. Matorral submontano, arroyos. Sierras de La Gavia y de La Paila en Coah. 
Apéndice 1. Continuación.

Caryophyllaceae

Arenaria ludens Shinners. Bosque de pino-encino, suelos pedregosos. Sierras del Carmen y Santa Rosa en Coah. y región de Trans-Pecos, TX.

Drymaria axillaris Brandegee. Matorral de halófitas en suelos yesosos y salinos. N de la Sierra de La Paila y Laguna del Rey en Coah.

Drymaria coahuilana (I. M. Johnst.) B. L. Turner (D. lyropetala I. M. Johnst. var. coahuilana I. M. Johnst.). Matorral de halófitas en suelos yesosos y salinos. Valle de Cuatro Ciénegas, valles al norte de Sierra de La Paila y Laguna del Rey en Coah. y NW de Chih.

Drymaria elata I. M. Johnst. Matorral de halófitas, suelo yesoso. SW de Coah.

Drymaria pattersonii B. L. Turner. Matorrales micrófilo y rosetófilo en suelos yesosos. E de Coah. (entre Monclova y Candela).

Drymaria subumbellata I. M. Johnst. Matorrales micrófilo y rosetófilo, suelos yesosos. Valles aledaños a la Sierra de La Paila y Laguna del Rey en Coah.

Drymaria suffruticosa A. Gray ex S. Watson. Matorrales micrófilo y rosetófilo, suelos yesosos. SW de Coah.

Paronychia albomarginata Core. Matorrales micrófilo y rosetófilo, en suelos pedregosos. $\mathrm{S}$ de Coah. $\mathrm{y}$ áreas adyacentes con N.L. (corredor Saltillo-Monterrey).

Chenopodiaceae

Atriplex acanthocarpa (Torr.) S. Watson var. stewartii (I. M. Johnst.) Henr. Matorral de halófitas en valles con suelos yesosos y salinos. Valle de Cuatro Ciénegas, Sierra de Las Delicias, lagunas del Rey y del Guaje en Coah. y E de Chih.

Atriplex monilifera S. Watson (Endolepis monilifera (S. Watson) Stand1.). Matorral de halófitas en valles con suelos salinos y yesosos. Laguna de Mayrán y Bolsón de Viesca en Coah.

Atriplex prosopidium I. M. Johnst. Matorral de halófitas en valles con suelos salinos y yesosos. Municipios de Cuatro Ciénegas y Monclova, y Laguna del Rey en Coah.

Meiomeria stellata (S. Watson) Standl. Matorral de halófitas en valles con suelos salinos y yesosos. Municipios de Cuatro Ciénegas, Monclova, Lagunas del Jaco y del Rey en Coah. 
Apéndice 1. Continuación.

Suaeda palmeri (Standl.) Standl. Matorral de halófitas en valles con suelos salinos y yesosos. Municipios de Cuatro Ciénegas, Monclova, Viesca y Laguna de Mayrán en Coah. y $\mathrm{N}$ de Zac.

Convolvulaceae

Bonamia multicaulis (Brandegee) House. Matorrales micrófilo y rosetófilo, suelos arenosos. Bolsón de Mapimí y Laguna del Rey en Coah., E de Chih. y NE de Dgo.

Ipomoea zimmermanii J. A. McDonald. Matorrales micrófilo, rosetófilo y submontano, laderas rocosas. Sierra de La Paila en Coah.

Crassulaceae

Echeveria turgida Rose. Matorrales micrófilo y rosetófilo, suelo pedregoso. Municipio de Viesca y Sierra de Las Delicias en Coah.

Lenophyllum weinbergii Britton. Matorral submontano, laderas rocosas. Sierra de La Paila en Coah.

Sedum diminutum (R. T. Clausen) G. L. Nesom \& J. A. McDonald (S. parvum Hemsl. var. diminutum R. T. Clausen). Bosque de pino-encino, laderas rocosas. Sierra de La Madera en Coah.

\section{Ericaceae}

Comarostaphylis polifolia (Kunth) Klatt ssp. coahuilensis Henr. Bosque de pino-encino y matorral submontano, suelo pedregoso. Sierras de Parras, de La Paila, de La Madera en Coah. y E de Chih. (Sierra de Almagre).

Euphorbiaceae

Bernardia myricifolia (Scheele) S. Watson var. incanoides M. C. Johnst. Matorrales micrófilo y rosetófilo, laderas rocosas. Sierras de Jimulco en Coah. y El Rosario en Dgo.

Cnidoscolus shrevei I. M. Johnst. Matorrales micrófilo y rosetófilo, laderas rocosas. Sierra de Jimulco en Coah. y El Rosario en Dgo.

Euphorbia crepitata L. C. Wheeler. Matorral de halófitas, suelo yesoso. Valle de Cuatro Ciénegas y puerto Ventanillas en Coah.

Euphorbia cressoides M. C. Johnst. Bosque de pino piñonero, suelo rocoso. Sierra de Jimulco en Coah. 
Apéndice 1. Continuación.

Euphorbia fruticulosa Boiss. Matorrales micrófilo y rosetófilo, suelos pedregosos y yesosos. Valle de Cuatro Ciénegas, sierras de Solís y de Las Delicias en Coah., Río Nazas en Dgo. y W de N.L.

Euphorbia ivanjohnstonii M. C. Johnst. Matorral submontano, suelo pedregoso. Sierra del Pino en Coah.

Euphorbia pinkavana M. C. Johnst. Bosque de pino piñonero, cañones y laderas rocosas. Sierra de La Madera en Coah.

Euphorbia scopulorum Brandegee var. inornata M. C. Johnst. Matorral de halófitas, suelos yesosos y calcáreos. W y centro de Coah. (municipios de Cuatro Ciénegas, Gral. Cepeda y Ocampo)

Phyllanthus fraguensis M. C. Johnst. Bosque de pino piñonero, laderas rocosas. Sierra de La Fragua en Coah.

Fabaceae (Leguminosae)

Acacia glandulifera S. Watson. Matorrales micrófilo y rosetófilo y bosque de pino piñonero, suelo pedregoso. Sierra del Pino, S de Coah. (Carneros), SE de Chih., NE de Dgo., W de N.L. y N de Zac.

Astragalus carminis Barneby. Bosque de pino-encino, en arroyos. Sierras de La Madera, del Carmen, Santa Rosa, del Pino, de La Encantada en Coah. y de Almagre en Chih.

Astragalus coahuilae M. E. Jones (Hamosa coahuilae (M. E Jones) Rydb.). Matorrales micrófilo y rosetófilo, suelos rocosos. Sierra de Parras en Coah. y NE de Zac.

Astragalus mollissimus Torr. var. nitens Barneby. Matorrales micrófilo, rosetófilo y submontano, suelos pedregosos. Sierra de Parras y Saltillo en Coah.

Astragalus pomphocalyx Villarreal \& Carranza. Matorrales micrófilo y rosetófilo, en arroyos. SE de Coah. y W de N.L.

Astragalus rupertii Villarreal \& Carranza. Matorral submontano, suelos pedregosos. Centro y S de Coah.

Caesalpinia sessilifolia S. Watson (Poinciana sessilifolia (S. Watson) Rose). Matorrales micrófilo y rosetófilo, laderas y valles pedregosos. Sierras de Las Delicias y de Jimulco en Coah., El Rosario en Dgo. y SW de Chih. 
Apéndice 1. Continuación.

Coursetia insomniifolia Lavin. Matorrales micrófilo y rosetófilo, laderas rocosas. Centro de Coah. (Ventanillas).

Dalea boraginea Barneby. Matorral submontano, laderas rocosas. Sierras del Carmen y Santa Rosa (Rincón de María) en Coah.

Dalea melantha Schauer var. pubens Barneby. Matorrales micrófilo, rosetófilo y submontano, laderas rocosas. Sierra de Jimulco en Coah.

Dalea neo-mexicana A. Gray var. megaladenia Barneby. Matorrales micrófilo y rosetófilo, laderas y valles. Sierras del Carmen y Santa Rosa en Coah., E de Chih., NE de Dgo. y región de Big Bend, TX.

Mimosa turneri Barneby. Matorrales micrófilo y rosetófilo, suelos derivados de rocas volcánicas. E y centro de Coah., W de N.L. y región de Trans-Pecos en TX.

Mimosa unipinnata Parfitt \& Pinkava. Matorrales micrófilo y rosetófilo, laderas rocosas. Municipios de Cuatro Ciénegas y Múzquiz en Coah.

Phaseolus plagiocylix Harms. Matorral submontano, laderas rocosas. Sierra de La Paila en Coah. y W de N.L.

Pomaria fruticosa (S. Watson) B. B. Simpson (Caesalpinia fruticosa (S. Watson) Fisher). Matorrales micrófilo y rosetófilo, laderas y valles pedregosos. Sierras de Las Delicias y Jimulco en Coah. y El Rosario en Dgo.

Senna monozyx (Irwin \& Barneby) Irwin \& Barneby (Cassia monozyx Irwin \& Barneby). Matorrales micrófilo y rosetófilo, lomeríos y faldas de sierras. Sierras Mojada, de La Madera y de La Paila en Coah.

Senna pilosior (J. Macbr.) Irwin \& Barneby (Cassia bauhinoides A. Gray var. pilosior J. Macbr.). Matorrales micrófilo y rosetófilo, laderas bajas y valles. Bolsón de Mapimí, W de Chih., NE de Dgo. hasta la región de Big Bend, TX.

Fagaceae

Quercus carmenensis C. H. Müll. Bosque de encino, laderas pedregosas. Sierra del Carmen en Coah. y Brewster Co. en TX.

Quercus coahuilensis Nixon \& C. H. Müll. Matorral submontano, laderas. Sierras de la Concordia, del Pino, de La Madera, de Jimulco en Coah., Sierra El Diablo en Chih. 
Apéndice 1. Continuación.

Quercus galeanensis C. H. Müll. Bosque de pino-encino, laderas. Sierra Madre Oriental, Sierra de Arteaga en Coah. y municipio de Galeana en N.L.

Quercus gravesii Sudw. Bosque de encino, laderas y valles. Sierras de La Gavia, de La Paila, del Carmen y de La Madera en Coah. y región de Trans-Pecos, TX.

Quercus hintoniorum Nixon \& C. H. Müll. Bosque de coníferas, vegetación subalpina, laderas altas de sierras. Sierra Madre Oriental, Sierra de Arteaga en Coah., municipio de Santiago y cerro El Potosí en N.L.

Quercus invaginata Trel. Matorral submontano y bosque de encino, laderas, cañones y arroyos. Sierras de La Paila, de La Gavia, de la Gloria y Pájaros Azules en Coah. y NW de N.L.

Quercus saltillensis Trel. (Q. carnerosana Trel.). Bosque de encino, laderas. Sierras de Arteaga, de la Concordia, de Parras, de La Paila, de Zapalinamé en Coah. y áreas adyacentes del W de N.L.

Fouquieriaceae

Fouquieria shrevei I. M. Johnst. Matorrales micrófilo y rosetófilo, en suelos yesosos. Bolsones de Mapimí y de Cuatro Ciénegas en Coah.

Gentianaceae

Eustoma barkleyi Shinners. Áreas perturbadas, orillas de corrientes de agua. S de Coah. y W de N.L.

Sabatia tuberculata J. E. Williams. Matorral de halófitas, en suelos yesosos cerca de los cuerpos de agua. Valle de Cuatro Ciénegas en Coah.

Hydrophyllaceae

Nama constancei J. D. Bacon. Matorrales micrófilo y rosetófilo, laderas y suelos rocosos o yesosos. W de Coah.

Nama cuatrocienegense G. L. Nesom. Matorral de halófitas, suelos yesosos. Valle de Cuatro Ciénegas en Coah.

Nama flavescens Brandegee. Matorrales micrófilo y rosetófilo, laderas rocosas y yesosas. S de Coah. y W de N.L. 
Apéndice 1. Continuación.

Nama havardii A. Gray (N. stewartii I. M. Johnst.). Matorrales micrófilo y rosetófilo, laderas arenosas o pedregosas. W de Coah., NE de Chih. y S de la región de Trans-Pecos, TX.

Nama johnstonii C. L. Hitchc. Matorrales micrófilo, rosetófilo y submontano, suelos rocosos. Sierras de La Fragua, de Parras y de Jimulco en Coah.

Nama marshii (Standl.) I. M. Johnst. Matorrales micrófilo y rosetófilo, laderas pedregosas. E y centro de Coah.

Nama serpylloides Hemsl. var. serpylloides. Matorral de halófitas, suelos salinos. Valles de Hermanas al $\mathrm{N}$ de Monclova y de Cuatro Ciénegas en Coah.

Nama serpylloides Hemsl. var. velutinum C. L. Hitchc. (N. s. var. confertum I. M. Johnst.). Matorral de halófitas, suelos yesosos. Centro de Coah.

Nama stenophyllum A. Gray \& Hemsl. Matorral de halófitas, suelos yesosos y salinos. SW de Coah. y SE de Chih.

Phacelia carmenensis B. L. Turner. Bosque de encino, laderas rocosas. Sierra del Carmen en Coah.

Phacelia gypsogenia I. M. Johnst. Matorral de halófitas, suelos yesosos. SW de Coah., SE de Chih. y W de N.L.

Phacelia marshall-johnstonii N. D. Atwood \& Pinkava. Matorral de halófitas, suelos yesosos. Valle de Cuatro Ciénegas en Coah.

Lamiaceae (Labiatae)

Hedeoma chihuahuense (Henr.) B. L. Turner (H. hyssopifolium A. Gray var. chihuahuensis Henr.). Matorral submontano, laderas rocosas. Sierra Santa Rosa en Coah. y NW de Chih.

Hedeoma johnstonii Irving. Matorral submontano y bosque de encino, laderas. Sierra del Carmen en Coah.

Hedeoma irvingii B. L. Turner. Matorral submontano, laderas pedregosas de cerros. Sierra Madre Oriental, municipio de Ramos Arizpe en Coah. y límites con el de Villa de García en N.L.

Hedeoma montanum Brandegee. Matorral submontano, laderas bajas pedregosas. Sierras de La Paila, La Fragua, Los Órganos y Los Remedios en Coah. 
Apéndice 1. Continuación.

Hedeoma oblatifolia Villarreal. Bosque de pino-encino, laderas rocosas. Sierra La Concordia en Coah.

Hedeoma palmeri Hemsl. var. santiagoanum B. L. Turner. Bosque de pino-encino, suelo rocoso. Sierra Madre Oriental, municipios de Arteaga en Coah. y Santiago en N.L.

Monarda pringlei Fernald (M. eplingiana Standl.). Matorral submontano y bosque de encino, laderas bajas y arroyos. Sierra del Carmen en Coah, hasta la Sierra Madre Oriental en N.L.

Poliomintha dendritica B. L. Turner. Matorral submontano, laderas y arroyos. Sierras de San Marcos en Coah. y de Lampazos en N.L.

Poliomintha maderensis Henr. Bosque de coníferas, laderas rocosas. Sierra de La Madera en Coah.

Salvia chamaedryoides Cav. var. coahuilensis (Fernald) K. M. Peterson (S. coahuilensis Fernald). Matorrales micrófilo y rosetófilo, suelos pedregosos. Sierras de Parras, de La Gavia y San Marcos en Coah. y W de N.L.

Salvia lanicalyx Epling. Matorral submontano, suelo rocoso. Sierra de Parras en Coah.

Salvia monclovensis Fernald. Bosque de pino-encino, cañones. Sierras de La Madera, de La Gavia y de La Gloria en Coah.

Salvia pseudopallida Epling. Bosque de coníferas, cañones. Sierra de La Madera en Coah.

Salvia purpusii Brandegee. Matorral submontano, laderas rocosas. Sierra Zapatero en Coah.

Satureja maderensis Henr. Bosque de coníferas, laderas rocosas. Sierra de La Madera en Coah.

Scutellaria carmenensis Henr. Bosque de pino-encino, laderas y cañones. Sierra del Carmen en Coah.

Scutellaria muzquiziana B. L. Turner. Bosque de pino-encino, laderas y arroyos. Sierras de La Gavia y de Santa Rosa en Coah.

Scutellaria stewartii B. L. Turner. Bosque de encino y de pino-encino, laderas. Sierras de La Madera, de La Paila y de La Gloria en Coah. 
Apéndice 1. Continuación.

Stachys hintoniorum B. L. Turner. Bosque de pino-encino, laderas rocosas. Sierra Madre Oriental, municipios de Arteaga en Coah., así como de Santiago y Galeana en N.L.

\section{Loasaceae}

Eucnide durangensis H. J. Thomps. \& A. M. Powell. Matorral submontano, laderas rocosas. Sierras de Jimulco en Coah. y El Rosario en Dgo.

Mentzelia mexicana H. J. Thomps. \& Zavort. Matorrales micrófilo y rosetófilo, suelos pedregosos. Sierras del Carmen y Santa Rosa en Coah., región de Big Bend y alrededores de Río Grande, TX.

Mentzelia pachyrhiza I. M. Johnst. Matorrales micrófilo y rosetófilo, suelos pedregosos. Sierras de Parras y de Jimulco en Coah., Bolsón de Mapimí, E de Chih., NE de Dgo. y región de Trans-Pecos en TX.

Mentzelia saxicola H. J. Thomps. \& Zavort. Matorrales micrófilo y rosetófilo, suelo pedregoso. W y S de Coah. (Sierra de Parras), E de Chih., NE de Zac. y W de TX.

Petalonyx crenatus S. Watson. Matorral de halófitas en dunas y suelos yesosos. Valle de Cuatro Ciénegas en Coah.

Malpighiaceae

Echinopterys setosa Brandegee. Matorrales micrófilo, rosetófilo y submontano, laderas rocosas. S y W de Coah. (sierras de La Madera y de La Paila).

Malvaceae

Abutilon coahuilae Kern. Matorrales micrófilo y rosetófilo, suelos arenosos y pedregosos. Centro y S de Coah. (sierras de Parras y de La Paila, así como Valle de Cuatro Ciénegas).

Batesimalva lobata Villarreal \& Fryxell. Matorral submontano, suelo rocoso. Sierras de Jimulco en Coah. y El Rosario en Dgo.

Sphaeralcea endlichii Ulbr. Matorrales micrófilo y rosetófilo, suelos arenosos y pedregosos. S y W de Coah. (sierras de Parras y de La Paila), SE de Chih., NE de Dgo., N de Zac. y W de N.L.

Sphaeralcea reflexa Fryxell, Valdés \& Villarreal. Matorrales micrófilo y rosetófilo, en suelos rocosos. Sierras de Jimulco en Coah. y El Rosario en Dgo. 
Apéndice 1. Continuación.

Martyniaceae

Proboscidea spicata Correll. Matorrales micrófilo y rosetófilo, suelos arenosos. Sierra del Carmen en Coah. y región de Trans-Pecos, TX.

Nyctaginaceae

Anulocaulis eriosolenus (A. Gray) Standl. Matorrales micrófilo y rosetófilo, suelos pedregosos. Valle de Cuatro Ciénegas y S de Coah., NE de Dgo., E de Chih. y región de Big Bend, TX .

Anulocaulis hintoniorum B. L. Turner. Matorrales micrófilo y rosetófilo, en suelos yesosos. Sierra de Parras en Coah.

Selinocarpus palmeri Hemsl. Matorral de halófitas, en suelos salinos. Laguna de Mayrán y municipio de Torreón en Coah.

Selinocarpus purpusianus Heimerl. var. marshii (I. M. Johnst.) Fowler \& B. L. Turner. Matorral de halófitas con suelo yesoso. E y centro de Coah. (Valle de Hermanas, cerca de Monclova).

Selinocarpus purpusianus Heimerl var. purpusianus. Matorral de halófitas en suelos yesosos. Valle de Cuatro Ciénegas, Laguna del Rey, municipio de Monclova (Valle de Hermanas) en Coah. y W de N.L.

Onagraceae

Oenothera sp. Bosque de encino, en áreas abiertas. Sierra de Santa Rosa (Rincón de María) en Coah.

Papaveraceae

Argemone fruticosa A. Gray. Matorrales micrófilo y rosetófilo, suelos arenosos. Sierras de Parras y del Venado, W del municipio de Torreón en Coah.

Polemoniaceae

Gilia purpusii Brandegee. Matorrales micrófilo y rosetófilo, lomeríos pedregosos. Municipios de Viesca y Matamoros en Coah.

Ipomopsis aggregata (Pursh) V. E. Grant. var. carmenensis Henr. Bosque de pino-encino, laderas rocosas. Sierra del Carmen en Coah. 
Apéndice 1. Continuación.

Ipomopsis wendtii Henr. Bosque de pino-encino, laderas rocosas. Sierra del Carmen en Coah.

Polygalaceae

Polygala maravillasensis Correll. Matorrales micrófilo y rosetófilo, suelos pedregosos. Sierra del Carmen y Santa Rosa, cañón del Río Bravo (Boquillas) en Coah., área adyacente en TX., y NE de Chih.

Polygala parrasana Brandegee. Matorral submontano, laderas rocosas en cañones. Sierras de Parras, de La Paila, Mojada y de Jimulco en Coah.

Polygala viridis S. Watson. Matorral submontano y bosque de encino, laderas rocosas. Sierras del Pino y de La Gloria en Coah.

Polygonaceae

Eriogonum hemipterum (Torr. \& A. Gray) S. Stokes var. griseum I. M. Johnst. Bosque de pino-encino, suelos pedregosos. Sierras del Pino en Coah. y El Diablo en Chih.

Eriogonum hemipterum (Torr. \& A. Gray) S. Stokes var. hemipterum. Bosque de pinoencino, suelos pedregosos. Sierras del Carmen y Santa Rosa en Coah. y las Montañas Chisos, TX.

Eriogonum henricksonii Reveal. Matorral micrófilo y rosetófilo, en suelos yesosos. Sierra Las Delicias en Coah.

Primulaceae

Samolus dichondrifolius Channell. Vegetación cercana a corrientes de agua en suelos yesosos. Sierra Las Delicias en Coah.

Samolus ebracteatus Kunth. var. coahuilensis Henr. Vegetación cerca de cuerpos de agua en suelos yesosos y dunas. Valle de Cuatro Ciénegas, Sierras de Parras y Las Delicias en Coah.

Ranunculaceae

Clematis coahuilensis Keil. Matorral submontano y bosque de pino-encino, cañones y lugares protegidos. Sierras de La Madera y de Jimulco en Coah. y El Rosario en Dgo., así como W de N.L. 
Apéndice 1. Continuación.

Rhamnaceae

Rhamnus standleyana C. B. Wolf. Bosque de pino-encino, en suelo arcilloso. Sierras de La Madera y de La Paila en Coah.

Rosaceae

Crataegus aurescens J. B. Phipps. Bosque de pino-encino, laderas. Sierra Madre Oriental, Jamé en Coah. y cerro de El Potosí en N.L.

Crataegus baroussana Eggl. var. baroussana. Bosque de pino-encino. Sierra de Zapalinamé en Coah. y cerro de El Potosí en N.L.

Crataegus baroussana Eggl. var. jamensis J. B. Phipps. Bosque de pino-encino, laderas. Sierra Madre Oriental, alrededores de Jamé en Coah.

Crataegus grandifolia J. B. Phipps. Bosque de pino-encino, laderas y valles. Sierra Madre Oriental, alrededores de Jamé en Coah.

Crataegus greggiana Eggl. var. pepo J. B. Phipps. Bosque de pino-encino, laderas. Sierra Madre Oriental, alrededores de Jamé, Serranías del Burro en Coah. y municipio de Galeana en N.L.

Crataegus johnstonii J. B. Phipps. Bosque de coníferas, laderas. Sierras del Carmen y Santa Rosa en Coah.

Crataegus sulfuracea J. B. Phipps. Bosque de pino-encino, laderas. Sierra Madre Oriental, Jamé en Coah. y cerro de El Potosí en N.L.

Crataegus tracyi Ashe ex Eggl. var. coahuilensis J. B. Phipps. Bosque de pino-encino, laderas. Sierra del Carmen en Coah.

Prunus cercocarpifolia Villarreal. Matorral submontano, suelo pedregoso. S de Coah.

Rosa woodsii Lindl. var. maderensis Henr. Bosques de coníferas y pino-encino, laderas y cañones. Sierra de La Madera en Coah.

Vauquelinia californica (Torr.) Sarg. ssp. retherfordii (I. M. Johnst.) Hess \& Henr. Matorral submontano, laderas pedregosas. Sierras de Jimulco en Coah. y El Rosario en Dgo. 
Apéndice 1. Continuación.

Rubiaceae

Chiococca henricksonii M. C. Johnst. Matorrales micrófilo y rosetófilo, laderas rocosas. Sierra de La Fragua y Sierra de Las Delicias en Coah.

Coutaportla pailensis Villarreal. Matorrales micrófilo y rosetófilo, laderas rocosas. Sierra de La Paila en Coah.

Galium carmenicola Dempster. Bosques de coníferas y encino, laderas y arroyos. Sierras del Carmen y Santa Rosa en Coah.

Galium mexicanum Kunth. var. flexicum Dempster. Bosques de encino y coníferas, laderas y arroyos. Sierras del Carmen y Santa Rosa en Coah. y Montañas Chisos en TX.

Hedyotis mullerae Fosberg (Houstonia mullerae (Fosberg) Terrell, Stenaria mullerae (Fosberg) Terrell). Matorral submontano, laderas y arroyos. Sierras de La Fragua, de San Marcos y de La Madera en Coah.

Hedyotis teretifolia (Terrell) G. L. Nesom. Matorrales micrófilo y rosetófilo, suelos yesosos. Sierra de Las Delicias y Sierra de Las Margaritas en Coah.

Machaonia pringlei A. Gray. Matorral submontano, laderas y arroyos. Sierra de Jimulco en Coah.

Rutaceae

Choisya katherinae C. H. Müll. Matorral submontano, laderas rocosas. Sierras Mojada, de La Madera y de Jimulco en Coah. y del Pulpito en Chih.

Thamnosma pailenis M. C. Johnst. Matorrales micrófilo y rosetófilo, laderas pedregosas y arroyos. Sierra de La Paila en Coah.

Thamnosma stanfordii I. M. Johnst. Matorral submontano, arroyos. Sierras de Jimulco, de Parras y Las Delicias en Coah.

Scrophulariaceae

Leucophyllum laevigatum Standl. var. griseum (I. M. Johnst.) Henr. (L. virescens I. M. Johnst.). Matorrales micrófilo, rosetófilo y submontano, suelos pedregosos. Sierras de Parras y de Carneros en Coah., $\mathrm{N}$ de Zac. y $\mathrm{N}$ de S.L.P. 
Apéndice 1. Continuación.

Leucospora coahuilensis Henr. Matorrales micrófilo, rosetófilo y submontano, suelos pedregosos y arroyos. Sierras Mojada, de Jimulco, de La Madera, de La Fragua, de Las Margaritas, de Los Alamitos y Bolsón de Mapimí en Coah., Sierra de El Diablo en Chih., Sierra de El Rosario en Dgo. y N de Zac.

Mabrya coccinea (I. M. Johnst.) Elisens. Matorrales micrófilo y rosetófilo, laderas pedregosas, suelos yesosos. Sierra de Las Delicias en Coah.

Penstemon henricksonii Straw. Bosque de pino-encino, cañones. Sierra de La Madera en Coah.

Penstemon luteus G. L. Nesom. Bosque de pino-encino, laderas rocosas. Sierra del Coahuilón en el municipio de Arteaga, Coah.

Penstemon punctatus Brandegee. Bosque de pino-encino y matorral submontano, arroyos. Sierra de La Paila en Coah.

Seymeria coahuilana (Pennell) Standl. (Afzelia coahuilana Pennell). Matorral submontano, laderas y cañones. Sierra de La Gloria en Coah.

Seymeria falcata B. L. Turner. Matorral submontano, laderas y cañones. Sierras de La Madera, del Pino, de La Fragua, del Carmen y Mojada en Coah., sierras El Diablo y Chupaderos en Chih.

Seymeria pailana B. L. Turner. Matorral submontano y bosque de encino, laderas y cañones. Sierras de La Paila y de La Gavia en Coah.

Solanaceae

Lycium arochae $\mathrm{F}$. Chiang, T. Wendt \& E. J. Lott. Matorrales micrófilo y rosetófilo, valles con suelos arenosos. Laguna Colorada cerca de Cuatro Ciénegas en Coah.

Lycium californicum A. Gray. var. interior F. Chiang. Matorral de halófitas, suelos salinos. W de Coah. y la confluencia entre los estados de Coah., Zac. y S.L.P.

Lycium leiospermum I. M. Johnst. Matorral halófito, suelos salinos. SE de Coah., NE de Zac. y $\mathrm{N}$ de S.L.P.

Lycium parishii A. Gray var. modestum (I. M. Johnst.) F. Chiang (L. modestum I. M. Johnst.). Matorrales micrófilo y rosetófilo, suelos salinos y yesosos. Centro y S de Coah. (Valle de Cuatro Ciénegas), W de N.L. y N de S.L.P. 
Apéndice 1. Continuación.

Solanum johnstonii M. D. Whalen. Matorrales micrófilo y rosetófilo, suelos arenosos y pedregosos. Sierras de Jimulco en Coah. y El Rosario en Dgo.

Verbenaceae

Tetraclea subinclusa I. M. Johnst. (T. coulteri A. Gray var. subinclusa (I. M. Johnst.) Moldenke. Matorral de halófitas, en suelos yesosos. Valle de Cuatro Ciénegas en Coah.

Zygophyllaceae

Fagonia scoparia Brandegee. Matorral de halófitas, en suelos yesosos. Valle de Cuatro Ciénegas y Sierra Las Delicias en Coah., NE de Dgo. y SE de Chih.

\section{LILIOPSIDA (MONOCOTYLEDONEAE)}

\section{Agavaceae}

Agave bracteosa $\mathrm{S}$. Watson. Bosque de pino-encino, laderas y paredes rocosas. Sierra Madre Oriental (Ramos Arizpe) y Sierra de La Gavia en Coah., W de N.L. (Santa Catarina y Santiago).

Agave montana Villarreal. Bosque de coníferas y vegetación subalpina, laderas rocosas. Partes altas de la Sierra Madre Oriental (límite entre Coah. y N.L.).

Agave parrasana Berger. Bosque de pino-encino, laderas rocosas. Sierras de Parras, de San Marcos, de La Gavia, de La Paila y Santa Rosa en Coah.

Agave scabra Salm.-Dyck ssp. maderensis Gentry. Matorral submontano y bosque de encino. Sierra de La Madera en Coah.

Agave victoriae-reginae Moore. Matorrales micrófilo y rosetófilo, laderas y paredes rocosas. Municipios de Ramos Arizpe, Torreón y Viesca en Coah., Cañón de la "Huasteca", municipio de Santa Catarina en N.L. y Sierra El Rosario en Dgo.

Dasylirion stewartii I. M. Johnst. Matorral desértico, laderas pedregosas. Sierra del Pino en Coah.

Yucca coahuilensis Matuda \& Piña. Matorral tamaulipeco, suelos arenosos y pedregosos. NE de Coah.

Yucca endlichiana Trel. Matorrales micrófilo y rosetófilo, laderas bajas y pedregosas. Valle de Cuatro Ciénegas y Sierra de La Paila en Coah. 
Apéndice 1. Continuación.

Yucca linearifolia Clary. Matorrales micrófilo y rosetófilo, laderas bajas. S de Coah. (municipio de General Cepeda) y W de N.L. (municipio de Galeana).

\section{Cyperaceae}

Scirpus coahuilensis Svenson. Bosque de pino-encino, orillas de arroyos. Sierra de La Fragua en Coah.

\section{Liliaceae}

Dandya purpusii (Brandegee) H. E. Moore (Muilla purpusii Brandegee). Matorrales micrófilo y rosetófilo, suelo pedregoso. Sierra de La Paila en Coah.

Hemiphylacus latifolius S. Watson. Bosque de pino piñonero, suelo calcareo. Sierra Madre Oriental (municipios de Saltillo y Arteaga) y Sierra de Jimulco en Coah.

Milla bryanii I. M. Johnst. Matorrales micrófilo y rosetófilo, laderas rocosas. Valle de Cuatro Ciénegas en Coah. y Sierra el Pulpito en Chih.

Orchidaceae

Malaxis wendtii Salazar. Bosque de pino-encino, laderas rocosas. Sierras del Carmen y de La Madera en Coah.

Poaceae

Bouteloua eriostachya (Swallen) Reeder. Matorrales micrófilo y rosetófilo, laderas rocosas. Sierras Cruces, de La Fragua, de Los Alamitos y Valle de Buenavista en Coah.

Bouteloua johnstonii Swallen. Matorrales micrófilo y rosetófilo, suelo yesoso y laderas bajas. Sierras de los Órganos, de La Fragua, Las Delicias, de La Paila y alrededores de Tanque La Luz en Coah.

Bromus densus Swallen. Bosque de pino-encino en valles y laderas. Sierra Madre Oriental, municipios de Arteaga y Saltillo en Coah. y Santiago en N.L.

Festuca coahuilana M. González-Ledezma \& S. D. Koch. Bosque de coníferas, laderas rocosas. Sierra de La Madera en Coah.

Festuca pinetorum Swallen. Bosque de coníferas, laderas rocosas. Sierra Madre Oriental, municipios de Arteaga en Coah. y Santiago en N.L. 
Apéndice 1. Continuación.

Festuca valdesii M. González-Ledezma \& S. D. Koch. Bosque de coníferas, laderas rocosas. Sierra Madre Oriental, Sierra de Zapalinamé en Coah. y áreas colindantes de N.L.

Sporobolus coahuilensis Valdés-Reyna. Matorrales micrófilo y rosetófilo, suelo yesoso. Valle de Cuatro Ciénegas y Sierra Las Delicias en Coah.

Sporobolus regis I. M. Johnst. Matorral de halófitas, suelo yesoso. Laguna del Rey en Coah.

Sporobolus spiciformis Swallen. Matorrales micrófilo y rosetófilo, suelos arenosos y yesosos. Valle de Cuatro Ciénegas, Laguna del Rey, Sierras Las Delicias y Viesca en Coah., NE de Dgo. y SE de Chih. 
Apéndice 2. Plantas endémicas para Megacoahuila y no registradas de Coahuila.

\section{Acanthaceae}

Mirandea huastecensis T. F. Daniel. Matorral submontano con suelo rocoso. La Huasteca en el municipio de Santa Catarina, N.L.

Siphonoglossa durangensis Henr. \& Hilsenb. Matorrales micrófilo y rosetófilo, partes bajas de las sierras. NE de Dgo. (Río Nazas).

Anacardiaceae

Rhus chiangii Young. Matorral submontano, suelo rocoso. Sierra El Rosario en Dgo.

Asteraceae

Strotheria gypsophila B. L. Turner (Graciela frankenioides Rzed.). Matorral de halófitas, suelos yesosos. San Roberto (municipio de Galeana) en N.L.

Cactaceae

Coryphantha longicornis Boed. Zacatal y matorrales micrófilo y rosetófilo, laderas rocosas. Río Nazas en Dgo.

Mammillaria guelzowiana Werderm. Matorrales micrófilo y rosetófilo, laderas rocosas. Río Nazas en Dgo.

Mammillaria pennispinosa Krainz var. nazasensis Glass \& Foster. Matorrales micrófilo y rosetófilo, laderas rocosas. Río Nazas en Dgo.

Caryophyllaceae

Drymaria pratheri B. L. Turner. Matorral de halófitas, suelo yesoso. Municipio de Mina, en el W de N.L.

Euphorbiaceae

Euphorbia correlli M. C. Johnst. Matorral de halófitas, suelo yesoso. Municipio de Galeana en N.L.

Fagaceae

Quercus graciliformis C. H. Müll. Bosque de encino, en cañones y orilla de corrientes de agua. Montañas Chisos, región de Trans-Pecos TX. 
Apéndice 2. Continuación.

Frankeniaceae

Frankenia johnstonii Correll ( $F$. leverichii B. L. Turner). Matorral de halófitas, suelos salinos y yesosos. W de N.L. y S de TX.

Frankenia margaritae Medrano. Matorral de halófitas, suelos salinos y yesosos. San Roberto (municipio de Galeana) en N.L.

Hydrophyllaceae

Nama turneri J. D. Bacon. Matorral de halófitas, suelos yesosos. Municipio de Galeana en N.L. y NE de S.L.P.

Polygonaceae

Eriogonum fimbriatum Hess \& Reveal. Matorrales micrófilo y rosetófilo, suelo yesoso. San Roberto (municipio de Galeana) en N.L.

Eriogonum turneri Reveal. Matorrales micrófilo y rosetófilo, suelo yesoso. San Roberto (municipio de Galeana) en N.L. 